\title{
Micro-Nanostructured Polymer Surfaces Using Injection Molding: A Review
}

\author{
K. Maghsoudi*, R. Jafari, G. Momen, M. Farzaneh \\ Department of Applied Sciences, University of Quebec in Chicoutimi (UQAC) \\ 555, boul. de l'Université, Chicoutimi, Quebec, G7H 2B1, Canada \\ *E-mail: Khosrow.maghsoudil@uqac.ca
}

\begin{abstract}
Micro injection molding is in great demand due to its efficiency and applicability for industry. Polymer surfaces having micro-nanostructures can be produced using injection molding. However, it is not as straightforward as scaling-up of conventional injection molding. The paper is organized based on three main technical areas: mold inserts, processing parameters, and demolding. An accurate set of processing parameters is required to achieve precise micro injection molding. This review provides a comparative description of the influence of processing parameters on the quality of final parts and the precision of final product dimensions in both thermoplastic polymers and rubber materials. It also highlights the key parameters to attain a high quality micro-nanostructured polymer and addresses the contradictory effects of these parameters on the final result. Moreover, since the produced part should be properly demolded to possess a high quality textured polymer, various demolding techniques are assessed in this review as well.
\end{abstract}

Keywords: injection molding; micro-nanostructure; processing parameters; demolding; replication

\section{Introduction}

Among the different polymer processing techniques used to produce materials having a specifically desired size and shape, injection molding is highly preferred for mass production systems, and has been used for many years in the polymer industry ${ }^{1-2}$. This technology is highly sought after for industrial applications due to: the low cost in the fabrication of polymeric parts especially for large quantities; versatile shapes; short cycle times; simple automation; and the possibility of simultaneous shaping of bulk and surface structures ${ }^{3-5}$.

Surfaces with micro-nanostructures and an aspect ratio (ratio of height to width) of more than 1:1 are used in a number of applications including antireflection coatings, antipollution and selfcleaning surfaces, cell culturing and differentiation, microlenses, bioinspired non-reflective, dry adhesion surfaces, and superhydrophobic surfaces 1-3, 6-11. Potential applications of these nanostructured surfaces are rapidly expanding. This popularity has led to studies that aim to use injection molding processes to produce microfeatured surfaces and as economically as possible 
12-13. Micro injection molding ( $\mu \mathrm{IM})$ technology is comprised of three different subcategories with regards to micro-sized phenomena involved:

I. Parts having a weight of milligrams or less;

II. Parts with microstructures less than $100 \mu \mathrm{m}$, created by replication using a micronanostructured mold;

III. Parts having tolerance in the micrometer range but without dimension limits ${ }^{14-16}$.

A general definition of injection molding of surfaces with micro-nanostructure can be considered as the production of parts with micro and/or nanostructure on its surface ${ }^{17}$. The critical aspect in the replication of micron or sub-micron features on the polymer surface is the necessary high precision involved ${ }^{5,18}$. Indeed, the precision of any replication depends on many parameters that may or may not be possible to control. Such parameters include feature size, shape and orientation relative to the filling flow direction, the distance of the features from the injection gate, and the aspect ratio. However, the most important parameters are the processing conditions of the selected injection molding process, e.g. mold temperature, melt temperature, holding pressure, injection velocity, holding time, and part thickness. There are many contradictions concerning the effect of these processing parameters, with mold temperature, holding pressure, and injection velocity being most important and having an inevitable influence on the quality of final replication.

Micro injection molding consists of steps similar to those of a conventional injection molding: filling, packing, holding, cooling, and demolding phases. The filling of cavities in conventional injection molding is, however, not as complex as filling microfeatured cavities [12]. In the micro injection molding process, the microstructures not only contribute to harden the polymer melt filling into the microstructures, but also increase the cooling rate of the material in the mold due to the increase of the surface to volume ratio. Therefore, proper filling of the material into the micro-nanostructures becomes more difficult ${ }^{19}$. Moreover, both a complete filling of a structure and its associated demolding are considered as the main factors for achieving a high quality replication ${ }^{3}$. Consequently, proper demolding is as important as appropriate filling.

It is very optimistic to assume that the melt materials may fill the microstructures, cool down, solidify, and are then ejected to produce a perfectly replicated structure on the surface ${ }^{3}$. Given that replication quality is highly influenced by mold temperature, melt temperature, holding pressure, and injection velocity, any small change in one of these parameters could markedly affect the quality of the replicated surface. Therefore, the filling process could be enhanced by optimizing the process conditions and demolding would be improved by using a low surface energy coating on the mold surface.

Various types of polymers are used to produce parts having microfeatured surfaces or micro components. As different polymers have various rheological, thermal, mechanical, electrical, and optical properties, polymers with the desired flow properties and a low viscosity at high temperatures are suitable as materials for microinjection molding ${ }^{20}$. A wide range of polymer materials have been studied ${ }^{19,21-26}$ including thermoplastic polymers such as polystyrene (PS) ${ }^{18}$, cyclic olefin copolymer $(\mathrm{COC})^{3,20,27-28}$, poly methyl methacrylate (PMMA) ${ }^{19,27}$, polypropylene 
(PP) ${ }^{19}$, polysulfone (PSU), polyoxymethylene (POM) ${ }^{29}$, polyethylene (PE) ${ }^{19}$, polyamide (PA)

${ }^{30-31}$, poly ether ether ketone (PEEK) ${ }^{32-33}$, polycarbonate $(\mathrm{PC})^{34-35}$, liquid crystal polymer (LCP) ${ }^{29}$, polybutylene terephthalate (PBT) ${ }^{36}$, acrylonitrile butadiene styrene (ABS) ${ }^{35}$, polyphenyl ether (PPE) ${ }^{37}$, and liquid silicone rubber (LSR) as a crosslinking polymer material ${ }^{38}$. To the best of our knowledge, only a few studies have been carried out on rubber materials ${ }^{38}$, a research area that our research group is actively investigating.

In the present review paper, three steps of the micro-nano injection molding process have essentially been considered. The first step is insert-making, where different kinds of materials used as inserts, considering their advantages and disadvantages. As the processing parameters have the most important role in injection molding, the effects of each processing parameter are thoroughly investigated with regards to the replication quality of the molded part, and contradictions are scrutinized. It has been tried to organize the effects of these parameters based on their positive or negative influence on the replication quality. It is noteworthy that final decision to set a series of processing parameters would depend on type of polymer material, geometry and dimensions and many other conditions in the process. Finally, the demolding methods, the challenges and solutions will be studied.

\section{Mold Inserts}

As tool making is expensive and time consuming, it is recommended for mass production. In the case of prototyping, using inserts could allow the industry to avoid multiple tool manufacturing. Consequently, the production of varied surface features would not be as expensive and time consuming ${ }^{39}$. An insert (also called a stamper or inlay) is an exchangeable cavity that is inserted into the mold. It extends the useful life of the tool and provides the possibility of changing and testing various structures and conditions using the main mold without the need to replace the complete mold and undergo expensive tool making process. Moreover, replacement of a worn insert is much less expensive than replacing a mold ${ }^{40}$. The use of interchangeable inserts also facilitates the creation of patterns on one or more of the cavity walls and provides micronanostructured mold surfaces ready to be replicated ${ }^{41}$. As the quality of the mold insert is higher, the success chance of the replication is more likely.

\subsection{Insert Material}

Inserts in the injection molding process can be fabricated from a number of potential inserts. Silicon is the conventional material because of the facility of silicon inserts fabrication. However, the convenience of using silicon is limited by its brittle behavior when subjected to the high pressure encountered during injection molding processes ${ }^{2,34,42}$. Thus, other materials may offer some advantages over the traditional use of silicon for inserts.

Metals such as nickel ${ }^{2-3,43-44}$, steel ${ }^{18,29,44}$, BMG (Bulk Metallic Glass) ${ }^{43-45}$, and aluminum ${ }^{46}$ represent other options for insert material, especially considering the ease of creating patterns via mechanical machining and electroforming of metals ${ }^{2}$.

Metallic inserts, however, increase the cooling rate of molten polymer due to their high thermal conductivity and diffusivity. This high cooling rate leads to the formation of a frozen skin layer 
between the polymer melt and the mold surface. Therefore, this non-melt skin layer does not let the molten polymer penetrate into the micro-nanostructures. The result is a poor replication ${ }^{1}$.

As such there are two options to circumvent this problem. The first is to apply a variotherm technique that takes advantage of the high-speed cooling and heating of the mold. This technique will be assessed later in section 3.1.1. The other option is to replace the metal insert with inserts composed of a thermally insulating polymer (heat retardation technique) that has a low thermal conductivity.

For this latter option, the inserts, composed of materials such as polymers, reduce the rate of cooling of the polymer melt. Accordingly, molten materials have much more time to fill the micro-nanostructures prior to solidifying. The most common polymers used for this purpose are PET, PUA ${ }^{4}, \mathrm{PI}^{39}, \mathrm{PVA}^{47-48}, \mathrm{PC}^{39}$, and PEEK ${ }^{33}$.

Use of polymeric inserts during both the packing and filling phases allows polymer melts to flow easily into the micropatterns. As shown in Fig.1, in the presence of a polymeric insert, varied in thickness, polymer melt had a slower decrease in temperature. Viscosity, as a function of temperature, remained low for a longer period and the fluidity of the polymer melt was significantly improved ${ }^{4}$.

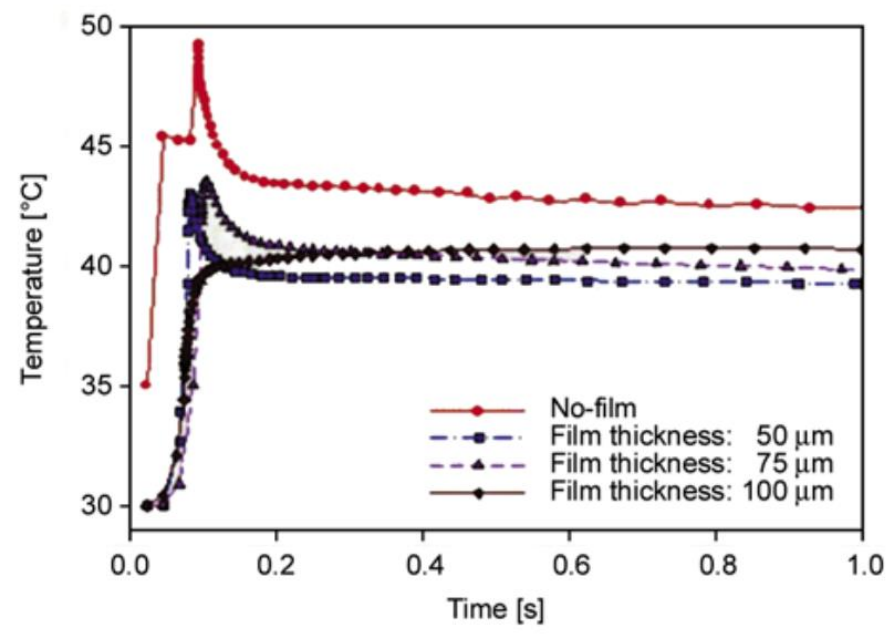

Fig.1. Changes in mold surface temperature over time, with and without a polymeric stamper ${ }^{4}$

Where a mold insert is deposited in only half the mold, an uneven cooling between the halves is observed ${ }^{14}$, possibly due to the different materials the mold and insert are made of. Therefore, it is recommended to use inserts that have similar thermal properties as the mold.

Ultra-violet cured poly(urethane acrylate) (PUA) is another possible insert material. Due to the formation of strong crosslinked chemical bonds upon curing, the UV cured PUA does not melt. It is considered as a Rigiflex or "rigid-yet-soft" insert having a relatively high rigidity of more than $40 \mathrm{MPa}$ and a low surface energy. As Rigiflex offers a combination of rigid and soft molds, they can be used instead of a hard or soft mold in many situations ${ }^{4,49}$. Park et al. ${ }^{4}$ used UV 
curable PUA resin and observed an effective delayed solidification near the mold surface. They also obtained desirable releasing characteristics that facilitated the demolding process.

Some polymeric materials, such as poly(vinyl alcohol) (PVA) ${ }^{47}$ and PDMS ${ }^{50}$, are used as inserts in hot embossing processes due to their being economical and offering effortless technique to create microfeatures. Regardless, the need remains for further investigation of these polymers to adapt them for injection molding process. It is, however, noteworthy that the polymeric inserts also deal with the same challenges as the metal inserts, i.e., proper filling ${ }^{39}$.

It is believed that during demolding, ejection forces can damage and/or deform the micronanopatterns. This problem will be discussed further in the demolding section of this review. A combination of film injection molding (FIM) and nanoimprint lithography methods is proposed to overcome this problem. In this method, PVA film is exploited as a sacrificing stamper. Benefitting from their water solubility properties, PVA films can be removed from the substrate material without serious difficulty. As a result, there is limited formation of the solid layer. However, in addition to PVA, any other polymer can be used if it is possible to be removed selectively with its special solvent ${ }^{47}$.

A number of materials other than metals and polymers, like silicon, quartz ${ }^{39}$, and gallium arsenide (GaAs), have been used ${ }^{34}$ with various achievements. Yoon et al. ${ }^{34}$ used silicon and GaAs wafers as inserts in a steel mold. To ensure that high pressure during the process did not damage the inserts, a 0.4-mm-thick polytetrafluoroethylene (PTFE) sheet was placed between the two aforementioned inserts.

The concept of hybrid inserts has been proposed ${ }^{39,51}$. Practically, a hybrid insert consists of both a polymeric and metallic layer. Epoxy, polyimide, polyether-ether-ketone are some materials used as the polymeric portion, and a metal protective layer is added to prevent any chemical interaction with the melt ${ }^{51}$. The low heat conductivity of the polymer layers improves filling as the cooling rate of the melt is reduced and the subsequent skin layer is avoided. When a hybrid insert is employed, the polymer melt temperature remains above the glassy state temperature $\left(\mathrm{T}_{\mathrm{g}}\right)$ for a longer period. This increases the likelihood that the molten polymer properly fills the structures before solidification. Fig.2 shows a successful filling using a hybrid insert in comparison with an incomplete filling in the case of a nickel insert ${ }^{39}$. 


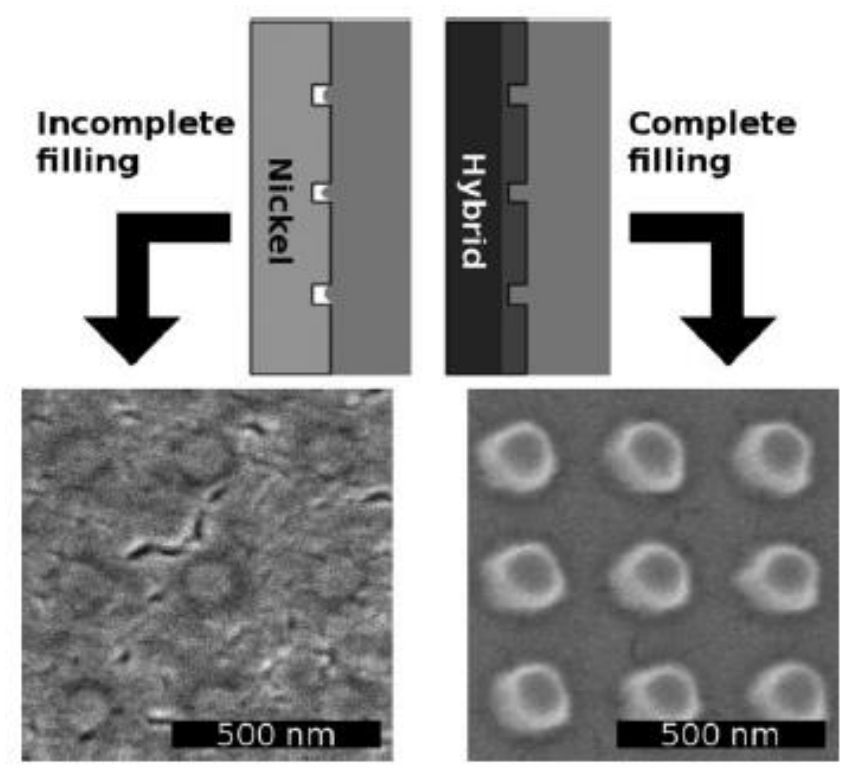

Fig.2. A successful filling using a hybrid insert compared to an incomplete filling using nickel insert ${ }^{39}$

Polyimide (PI) films coated with aluminum (Al) and polycarbonate (PC) have been assessed as insert materials. PI films showed outstanding replication of microstructures while undergoing more than 1000 cycles. However, PC inserts would deform as they could not endure the heat transferred from the polymer melt ${ }^{39}$.

Hybrid inlays can also lead to a stretching effect with nanopillars stretched up to $40 \%$ more than their expected height and thus nanopillars that are taller than the depth of the designed insert (Fig.3). Therefore, hybrid inserts provide a better opportunity to produce micro-nanostructured surfaces having a favorable replication quality ${ }^{39}$.

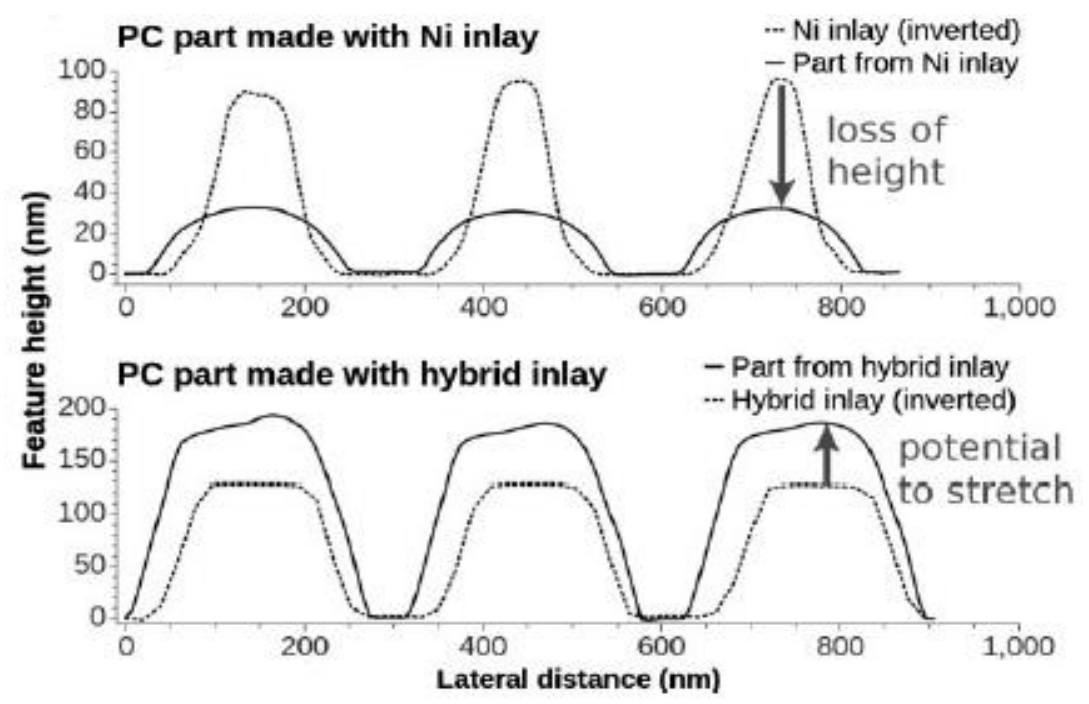

Fig.3. Comparison of molded PC feature heights made with a Ni inlay and a hybrid inlay ${ }^{39}$ 


\subsection{Insert Fabrication Methods}

Micro-nanometric dimensioned mold inserts require particular methods to be produced. These methods include (I) LIGA based (lithography, electroplating, molding) technologies (LIGA, UVLIGA, IB-LIGA, EB-LIGA) ${ }^{52-56}$; (II) 3D micro machining that regroups micro electrical discharge machining $(\mu \mathrm{EDM})$, micro mechanical milling and electrochemical machining $(\mathrm{ECM})$ using ultra-short pulses ${ }^{12,44,57}$; (III) silicon wet etching ${ }^{58}$; (IV) deep reaction ion etching (DRIE) ${ }^{1}$; (V) thick deep UV resists; (VI) laser ablation ${ }^{51,57,59-62}$, (VII) plasma treatment ${ }^{1,63-66}$, microdrilling and micro-turning ${ }^{67}$.

As an example of the chain of insert manufacturing ${ }^{43}$, Fig. 4 presents the manufacturing of a $\mathrm{Ni}$ insert using UV-LIGA process and the fabrication of a BMG insert using the thermoplasting forming process. The process chain is described in Fig. 4 and more details are available in ${ }^{43}$.

(a) (1)

(2)

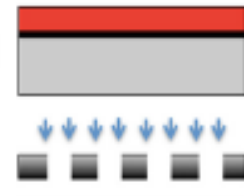

(3)

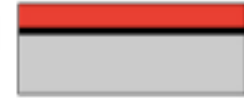

(4)

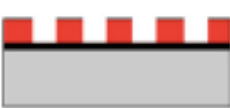

(b)

(1)

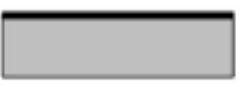

(2)

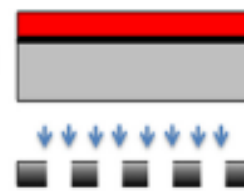

(3)

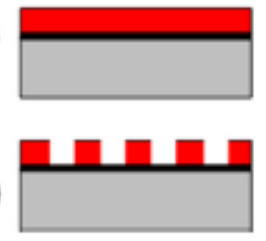

(5)

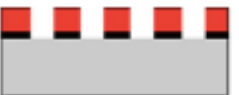

(6)

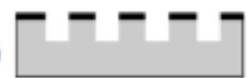

(7)

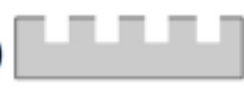

(8)

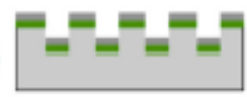

(9)

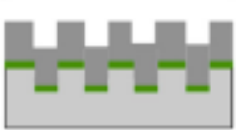

(5)

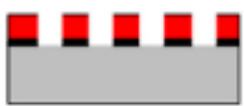

(6)

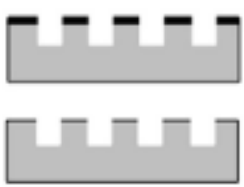

(8)

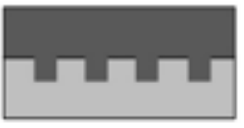

(9)

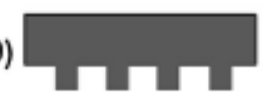

(10)
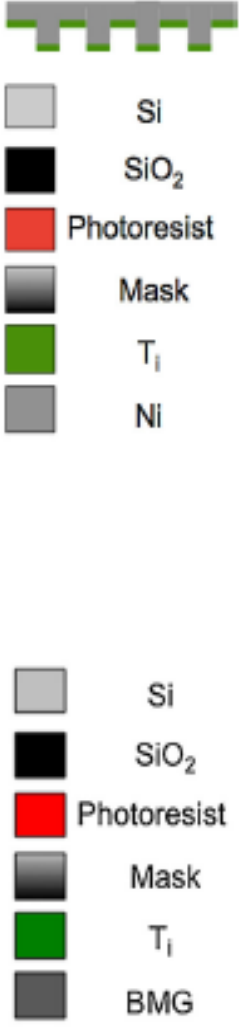

Fig.4. (a) UV-LIGA process: (1) Si oxidation, (2) spin coating photoresist, (3) UV lithography, (4) development,(5)etchingSiO2 and removing photoresist, (6) RIE etching of Si, (7) PVD coating Ti and Ni, (8) electroplating, (9) Si dissolving, (10) Ni wafer dicing and polishing; (b) thermoplastic forming process: (1)-(7) are the same as with the UV-LIGA process, (8) BMG thermoplastic forming into Si master, (9) Si dissolving in $\mathrm{KOH}$ solution ${ }^{43}$.

The $\mu$ EDM method is used to create mold inserts using high temperature metals or alloys. A submerging of the anode-cathode system into a dielectric fluid is necessary prior to the procedure. Metal removal begins by applying a high-voltage current between the cathode tool and anodic electrode. This method can be employed to fabricate high strength metals having a 
strength of more than $2000 \mathrm{MPa}$. As such it can be a suitable alternative for the manufacturing of mold inserts 57 .

Fig. 5 illustrates a die-sinking EDM process. First, a fluidic microfeature is milled into the surface of a graphite/copper electrode. Then, a stainless steel workpiece is eroded using a thermal process under a controlled electric spark ${ }^{44}$.

\section{Graphite/Cu}
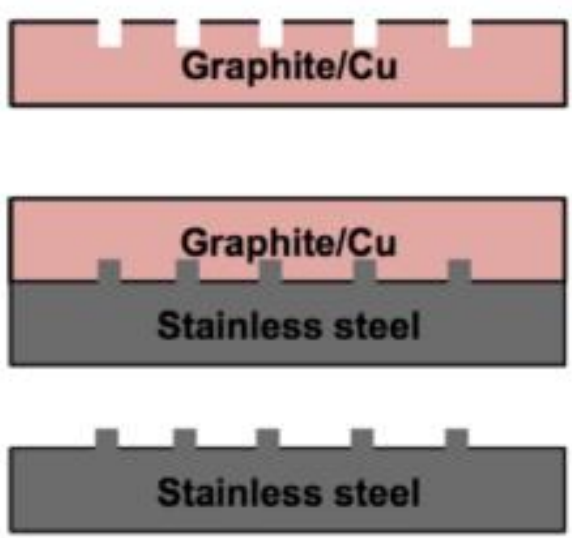

Fig.5. Micromilling of graphite/copper electrode, die-sinking EDM of stainless steel workpiece ${ }^{44}$

Laser ablation or laser milling has been considered as a reliable technique to produce inserts out of engineering materials ${ }^{59}$. This method relies on a high-intensity and concentrated laser beam that focuses and evaporates material at the focal point. The desired geometry can be achieved by moving either the substrate or the laser beam in $\mathrm{x}$ and/or $\mathrm{y}$ directions (see ${ }^{68}$ and ${ }^{69}$ for more details.). Fig.6 shows an ablated hole using laser beams showing the wall roughness and the debris ${ }^{60}$.

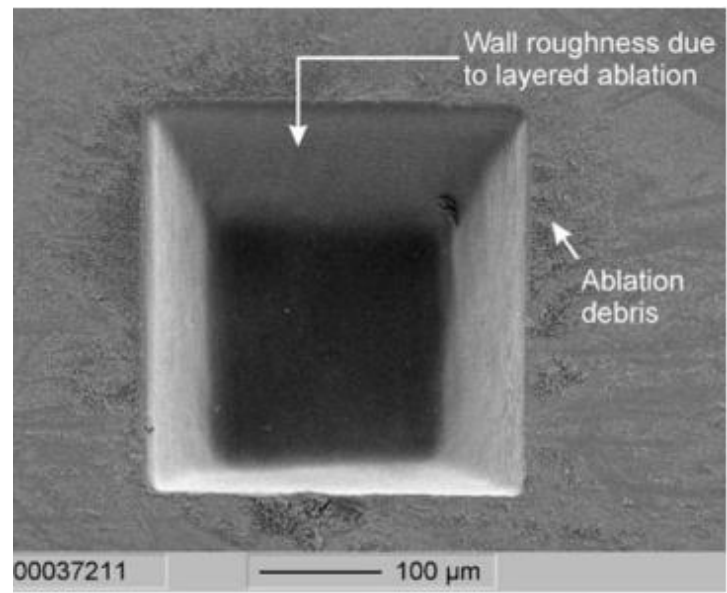

Fig.6. A laser-ablated structure showing the surface roughness and the debris produced from the process 60 
Laser milling joining with 3D CAD/CAM techniques is used to produce a shark skin-like surface on stainless steel inserts (see Fig.7) ${ }^{59}$. The laser milling process is accomplished by a series of random hatching inside each layer and a clear demarcation of edges. This leads to a favorable surface integrity and edge definition.

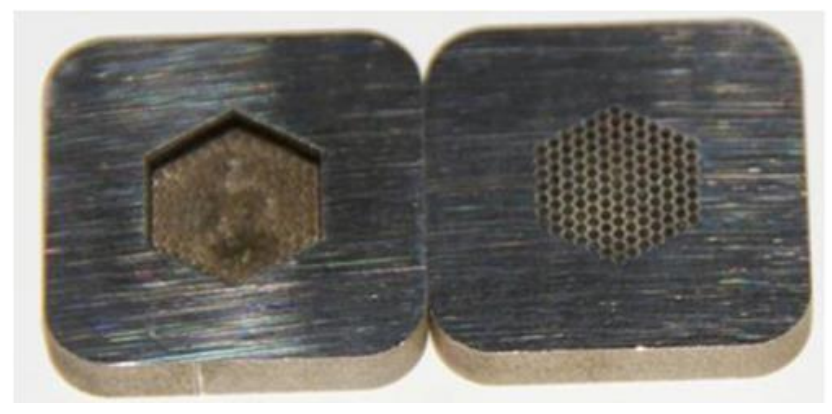

Fig.7. Shark skin-like structures machined by ms laser ablation ${ }^{59}$

\subsection{Types of Micro-Nanostructures}

Various types of micro-nanostructures have been used including micro-nanochannels with different cross sections e.g. cylindrical, rectangular ${ }^{45,55,70-72}$, micro-nanopillars ${ }^{5,18,32,39,73-74}$, micro-nanohairs ${ }^{75-76}$, and square meshes ${ }^{77}$.

It is well-known that polymer melts have very different flow behaviors in presence of microscale and macro-scale shapes ${ }^{78-79}$. The viscosity of a polymer melt increases markedly when dealing with microstructures. Young et al. ${ }^{72}$ modeled the filling distance into cylindrical microchannels, taking into consideration numerous assumptions including uniform insert temperature, constant heat transfer coefficient in the interface of the insert wall, and polymer melt. Based on the derived rheological model, the effect of channel radius on filling distance-penetration distance of the polymer melt into the microstructure-and aspect ratio of microfeatures (Fig.8 andFig.9). At a constant temperature, an increase in channel radius significantly increases the filling distance and filling velocity. There is a marked reduction in the cooling rate due to the decrease in the surface area to volume ratio. 


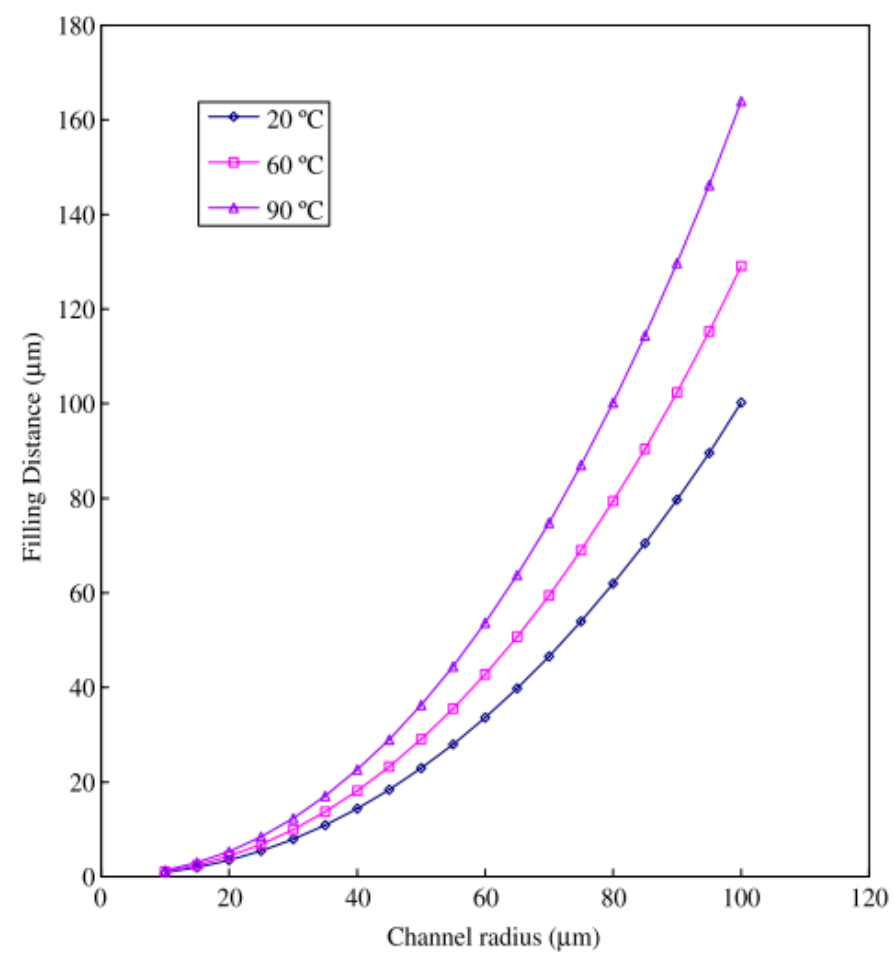

Fig.8. Filling distance in the microchannels having different radii and mold temperatures ${ }^{72}$

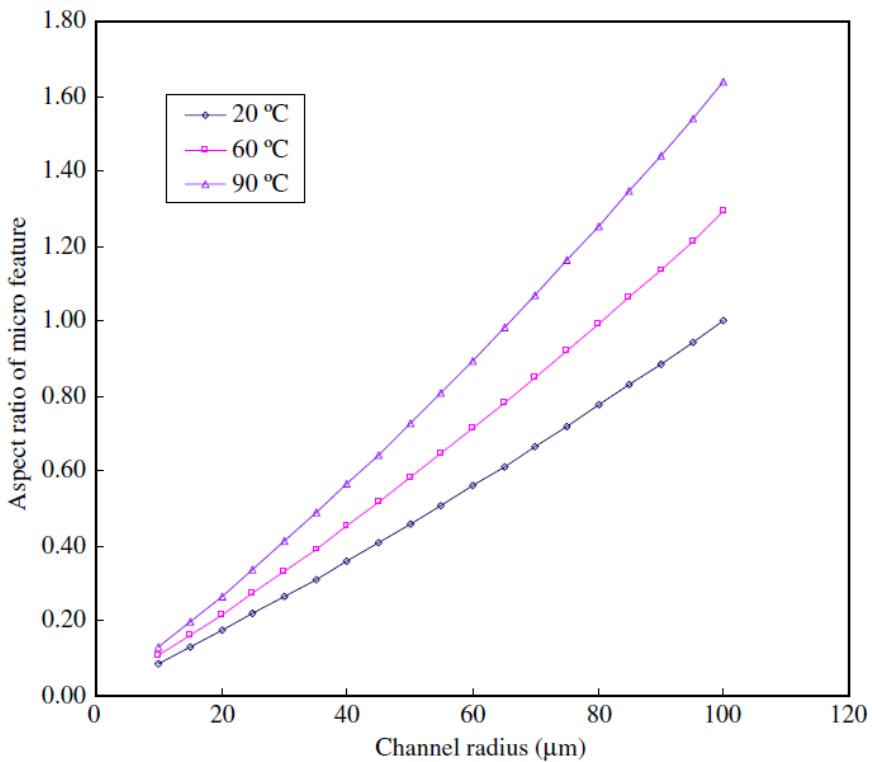

Fig.9. Aspect ratio of microfeature versus different channel radii and mold temperatures ${ }^{72}$

When comparing the specific surface area $\left(\mathrm{m}^{2} / \mathrm{m}^{3}=\mathrm{m}^{-1}\right)$ of different structures, the filling capacity of microchannels (triangle and rectangular cross sections) was most difficult relative to all other shapes, according to Fig.10. The filled length of circular structures was the greatest due to less specific surface area. 


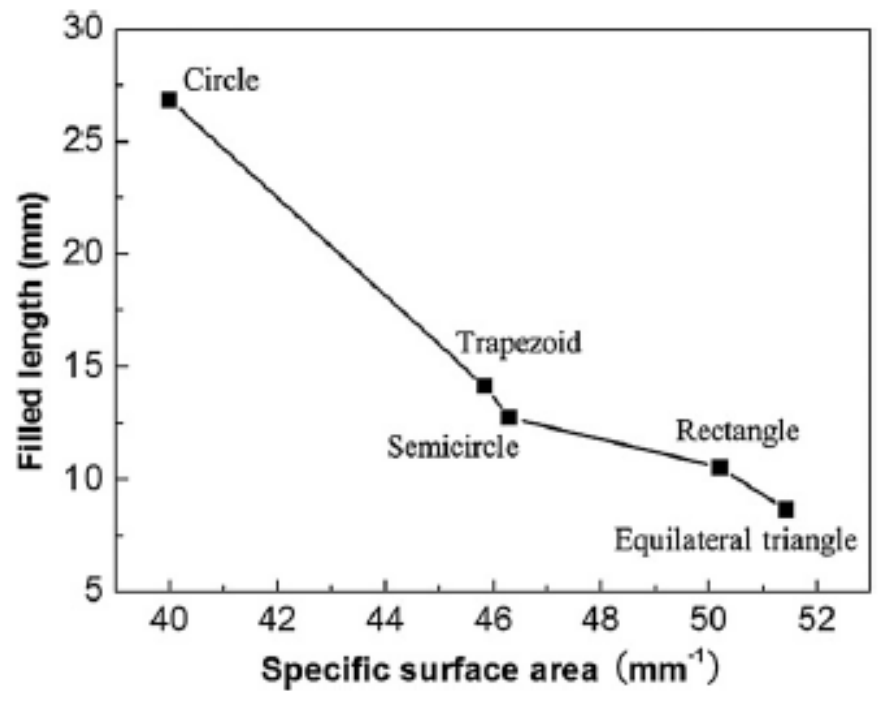

Fig.10. Filled length of different specific surface area (shape) $)^{14}$

Channels orientated parallel to the main flow direction favor a more accurate and desirable filling in comparison channels having a perpendicular orientation ${ }^{80}$. A proper design of features could lead to a higher water contact angle. For cone-shape structures, as the base diameter of the individual cones is reduced, the distances between the cone tips are lower and, as such, the contact angle increases ${ }^{38}$. However, the peak-to-peak distance should not decrease indiscriminately, as the formed compact surface would cause the drops spreading out more on the surface.

An arbitrary increase in the aspect ratio has a detrimental effect on replication quality ${ }^{14}$. As the aspect ratio of the features increases, due to greater polymer melt solidification, the replication percentage decreases. Fig.11 demonstrates this for a determined processing condition for injection molding of PMMA ${ }^{81-82}$. Undoubtedly, an optimal design of aspect ratio could result in both appropriate filling and an anticipated replication. 


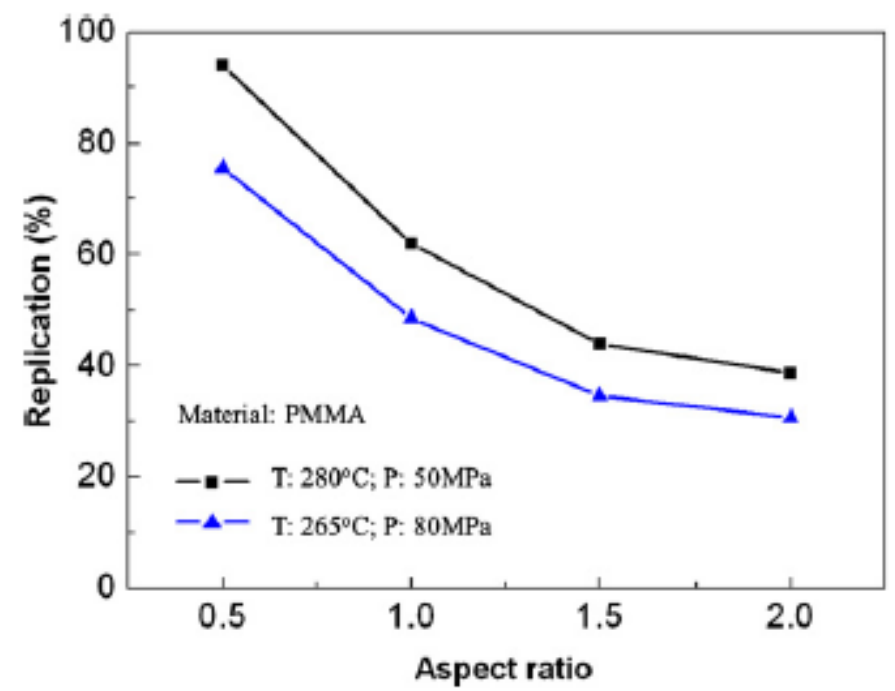

Fig.11. Effect of aspect ratio on replication percentage with various temperatures $(T)$ and packing pressures $(P)^{81}$

\subsection{Insert Durability}

Although studies of the durability of the produced surface structures undergoing wearing are very common, the durability of the inserts is also of great importance in micro-nano injection molding. If an insert is not acceptably robust after several molding cycles, there is little justification for using the insert.

In general, relative conventional hard inserts such as steel that has a superior strength, the durability of polymeric inserts can be problematic ${ }^{4}$. Some have suggested that instead of using plastic polymers (e.g. PEEK), crosslinked polymers such as the photocurable epoxy can provide much greater durability during the replication process. They also favor a greater precision in design precision during the fabrication ${ }^{39}$.

Stormonth-Darling et al. ${ }^{39}$ ran a single hybrid insert through more than 2000 cycles and observed no remarkable signs of wear and tear. Although AFM analysis showed a decrease in the features' depth over time, the reduction in depth was attributed to polymer residue build-up at the bottom of features. After cleaning with N-methyl-2-pyrrolidone (NMP), the average feature depth returned to its initial value.

In a test of the durability of BMG inserts ${ }^{45}$, a number of scratches on the insert surface were observed after 10000 molding cycles, although marked cracking was absent. However, after 20 000 molding cycles, a remarkable number of cracks had appeared on the insert surface. The roughness of the BMG inserts had increased about 10 fold after 20000 molding cycles relative to 10000 cycles.

The durability of silicon wafers as a potential insert material has been questioned because of its brittleness. Comparing the fracture toughness $\left(\mathrm{K}_{\mathrm{IC}}\right)$ of a silicon wafer with that of metals $(0.95$ $\mathrm{MPa} \cdot \mathrm{m}^{1 / 2}$ and $15-150 \mathrm{MPa} \cdot \mathrm{m}^{1 / 2}$, respectively) can be misleading as most ceramic materials show 
a high compressive strength rather than high fracture toughness ${ }^{34}$. Therefore, some argue that that silicon can be a promising material to fabricate inserts if it is manipulated properly.

A deposited metallic coating having a similar thermal expansion coefficient as a silicon wafer could be feasible to improve the compressive durability of the silicon tooling surface. The inserted silicon withstood injection pressure of $50 \mathrm{MPa}$. Moreover, a PTFE sheet was used to reinforce the silicon wafers produced acceptable results even after 3000 cycles ${ }^{34}$. In general, use of a hard coating material on a mold insert has been proposed to insure greater durability and protection of the insert's surface against wear ${ }^{31,83-84}$.

Consequently, in order to have accurate replication, it is necessary to have adequate filling, improved demolding, high quality surface, more durable insert material, as well as proper feature design and fabrication technique. After selecting the appropriate insert, the most fundamental step in micro-nano injection molding is determining the parameters of processing.

\section{Processing Parameters}

The molding process begins with the flow of molten polymer into the cavity, driven by the pressure applied by the screw. There is low flow resistance until the molten polymer reaches the microfeatures. At the edge of the microfeatures, resistance increases significantly and the polymer hesitates to fill the microstructures. Higher pressure is required to overcome this resistance and force the molten polymer down into the microfeatures ${ }^{18}$.

During the filling stage, due to the very fast heat loss of the molten polymer once in contact with the cavity wall, a solidified premature layer can quickly form. As soon as this solid layer is formed, there is not enough back pressure to push this layer into the structures and, consequently, the polymer melt is prevented from easily filling the micro-nanostructures. To avoid this problem, a number of solutions have been proposed including increasing the mold temperature and injection velocity as well as increasing both the injection and holding pressure ${ }^{85}$.

It should be noticed that the replication process can be continued until the packing phase, in addition to the filling phase ${ }^{3-4}$. Although the pressure applied to the melt during the first moments of contact between the molten polymer and the mold surface is negligible, it can increase during the packing phase and push the polymer melt into the micro-nanostructures ${ }^{3}$. This is only contingent on the polymer melt temperature. If the temperature is below the polymer $\mathrm{T}_{\mathrm{g}}$, increasing the pressure during the packing stage has almost no effect on the replication. In this case, the polymer melt has little time to flow, which is due to the formation of a so-called "skin-layer" on the surface of the polymer melt where it touches the cold cavity ${ }^{3,73}$.

As concerns the study of the microfeatures, both average height and the uniformity of the micronanostructure are also important. In addition to the high holding pressure and high mold temperature, an even distribution of cavity pressure is also required to achieve the highest level of uniformity and uniform height for microfeatures. These could be obtained by using efficient rapid heat cycle molding techniques and a thicker substrate ${ }^{86}$.

Injection velocity is the most questionable parameter among the other ones. Injection velocity is the speed at which polymer is injected into the mold cavity during the injection phase. Some 
argue that high injection velocities lead to better replication whilst others maintain an inverse relationship between injection velocity and replication quality ${ }^{86}$.

A means of ensuring the efficient filling and more economic process design, it is suggested that the micro injection could be first accomplished by using the conventional processing parameters, and then by using elevated temperature and pressure to ensure a high filling ratio ${ }^{87}$. However, this technique is highly questionable in the case of rubber injection molding. The mold temperature in the latter process is much lower than that for plastic injection molding. As such, in the rubber injection molding process, increasing the temperature after the filling stage leads to an increase in crosslink density and crosslinking reaction rate. This is highly undesirable especially in terms of micro-nanostructure filling.

Consequently, to achieve a complete and preferable replication, a combination of different processing parameters is necessary. A design of experiments (DoE) method has been used in a number of studies $18,20,28,86,88$. Through a DoE method, experimental data is acquired in a controlled way; the significant and non-significant factors affecting a process are determined, and, finally, the behavior of injection molding of micro-nanostructures can be carefully monitored. Based on ANOVA (analysis of variance) results, all of the main processing parameters have been shown to be significant including mold temperature, injection velocity, and holding pressure ${ }^{86}$. To better understanding the effect of various processing parameters on the replication quality and to help finding the optimum conditions, a process window could be provided $^{19}$.

\subsection{Mold Temperature}

Increasing mold temperature is generally considered as the most useful and practical way to improve the filling quality of the microstructures having a high aspect ratio $18,20,39,53,86,89$. This is due, mainly, to the easier melt flow in the cavity at higher temperatures due to the greater decrease of plastic polymer viscosity. Mold temperatures should be equal or greater to the softening temperature of the polymer used during the process [4]. The desired degree of filling will only be obtained if the polymer melt has enough fluidity to efficiently fill the microstructures. This is acquired only if the temperature of the polymer melt is above its $\mathrm{Tg}$ [4].

Since most filling of the micro-nanostructure takes place at the packing stage, it is crucial to keep the polymer melt temperature high enough at this stage. Increasing the mold temperature is only

effective when the temperature is above the polymer $\mathrm{T}_{\mathrm{g}}{ }^{18}$. When the mold temperature is equal to $\mathrm{T}_{\mathrm{g}}$ of the polymer, the acquired aspect ratio is 1 . This aspect ratio is not ideal for achieving micro-nanostructures. At higher mold temperatures i.e., the temperatures above $T_{g}$ of the polymer, the acquired aspect ratio is more acceptable ${ }^{3}$.

$\mathrm{Su}$ et al. ${ }^{42}$ found out that due to the high surface-to-volume ratio in the micro-nanostructured cavity, the temperature of molten polymer abruptly decreased immediately after the polymer entrance into the cavity, favoring the formation of the skin layer. Therefore, keeping the mold temperature high enough could eliminate any skin layer formation. Based on simulations, they recommended that the mold temperature about 30 to $40{ }^{\circ} \mathrm{C}$ above the polymer $\mathrm{T}_{\mathrm{g}}$ to guarantee an 
efficient filling and no solidified layer. Fig.12 shows the simulation results of acquired depth to opening ratio $(\mathrm{h} / \mathrm{d})$ versus radial location with various mold temperatures. The aspect ratio does not depend on the radial location when the temperature is high enough $\left(200{ }^{\circ} \mathrm{C}\right)$. Indeed, high mold temperature inhibits the fast cooling and would consequently lead to eliminating the formation of a frozen layer so that the microfeatures are filled efficiently ${ }^{20,53,73}$.

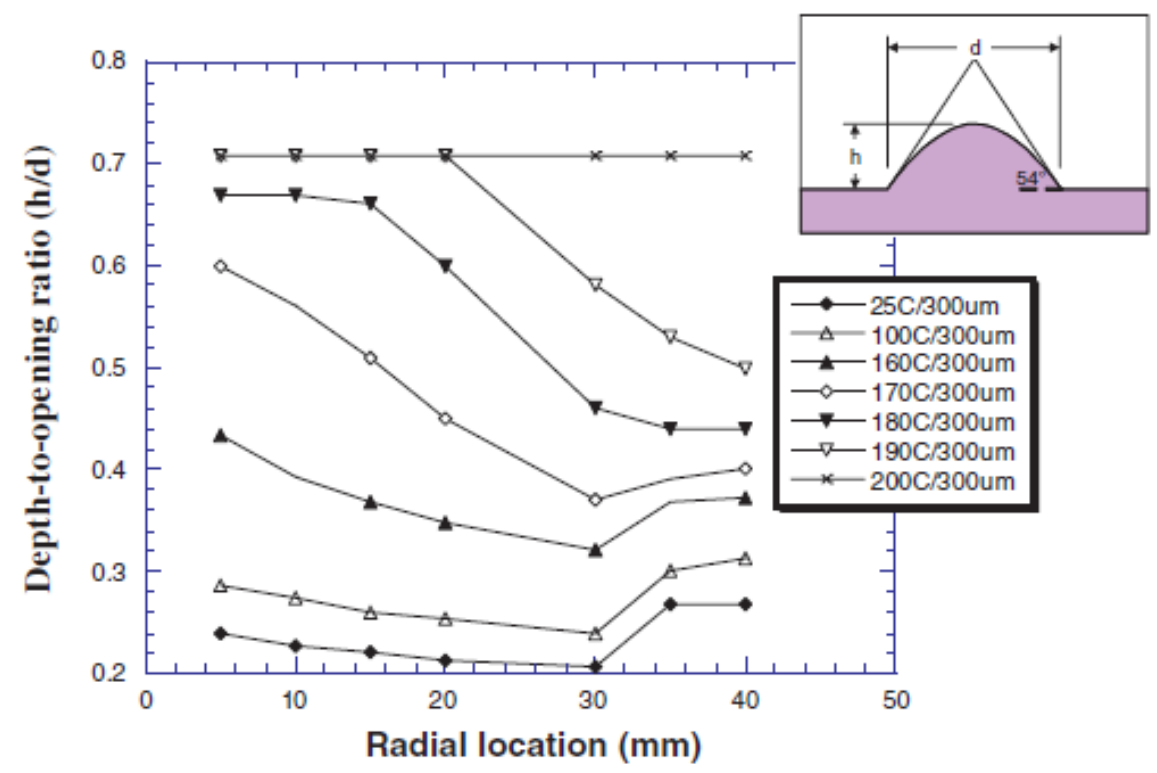

Fig.12. Simulation results of molding quality versus radial location with various mold temperatures ${ }^{42}$

The necessity of increasing the mold temperature to decrease the polymer viscosity has been discussed rheologically ${ }^{19}$. Based on two basic rheological equations (Eq. 1 and 2) for polymer materials, as the thickness of cavity $(h)$ decreases, the required shear strain rate $\left(\dot{\gamma}_{a v}\right)$ increases to keep the average velocity $\left(v_{a v}\right)$ constant.

$\dot{\gamma}_{a v}=2 v_{a v} / h$

Eq. 1

$\tau=\eta \dot{\gamma}$

Therefore, considering the classic stress-strain equation (Eq. 2) indicating the relation among shear stress $(\tau)$, polymer viscosity $(\eta)$ and shear strain $(\dot{\gamma})$, shear stress in the cavity increases with an increase in shear strain and thus, a higher filling resistance for the polymer. As a result, mold temperatures greater than glass temperature of the polymer could fulfill requirements for a successful replication.

It has been clearly shown that there is a direct relation between the acquired aspect ratio of the micro-nanostructures and the mold temperature. As the mold temperature increases, the achievable aspect ratio could increase ${ }^{15,90}$. Fig. 13 shows the effect of mold temperature and also micro-wall thickness on the replicated aspect ratio. 


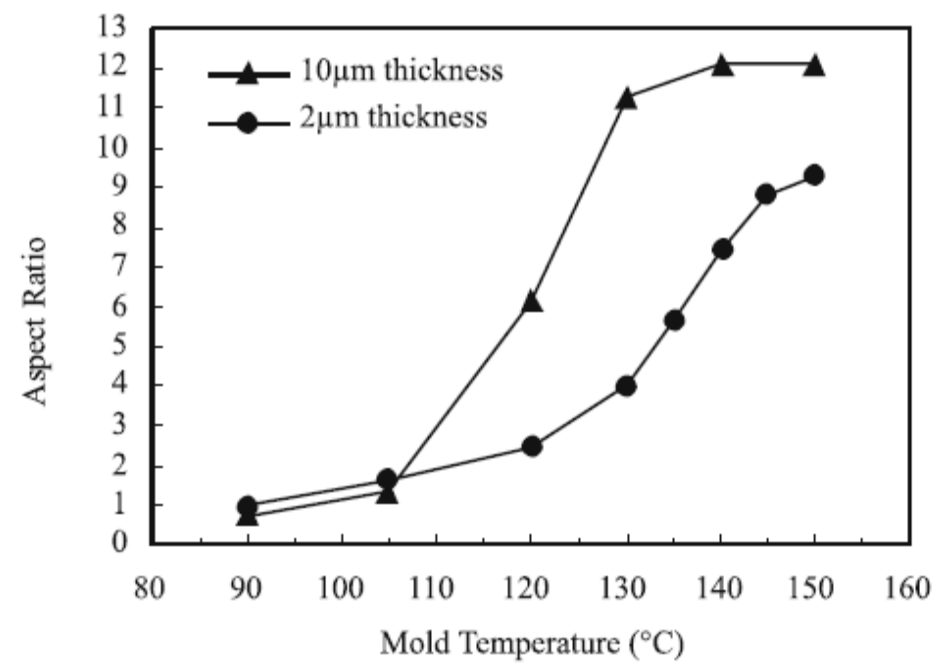

Fig.13. Effects of mold temperature on the achievable aspect ratio of the micro-walls with two different thicknesses ${ }^{19}$

Although it is asserted that the polymer viscosity is influenced by the mold temperature and the injection velocity more than any other processing parameter ${ }^{73}$, increasing the mold temperature permits decreasing both injection pressure and injection velocity ${ }^{42,91}$. In other words, if in an injection system, the mold temperature is high enough, it could be anticipated that injection pressure and injection velocity would not need to be increased.

Mold and melt temperature have been considered as the main parameters affecting micro-nano pattern quality, however only mold temperature affects the pattern height. A $50 \%$ increase of the mold temperature can lead to $>300 \%$ increase in the average height of micropillars ${ }^{18}$. Consequently, in the packing stage, the polymer melts having a lower viscosity can fill the micro-nanostructures more easily and efficiently. The improvement of height replication by increased mold temperature is because of two phenomena. i) reduced viscosity due to the increase of the mold temperature; and ii) the limitation of elastic effects during the filling and subsequent demolding stage. In other words, in the case of high injection velocity, the induced shear stress could lead to an elastic relaxation after removal of the manufactured part from the mold. An increase in mold temperature could prevent this undesirable induced elastic relaxation and cause a viscosity decrease without the development of an elastic effect ${ }^{41}$.

However, in addition to the positive effects of a mold temperature increase, there are some negative effects that require discussion. This includes an increase in cooling time and consequent increase in cycle times, an undesirable effect for industrial purposes ${ }^{23,53}$. Moreover, at higher mold temperatures, high adhesive and/or frictional forces lead to ineffective demolding of the micro-nanostructures, so that the replication quality deteriorates ${ }^{3}$. Excessive increases in mold temperatures can result in polymer degradation ${ }^{42,52}$.

Liou et al. ${ }^{19}$ demonstrated that there is a limit for increasing mold temperature. A SEM micrograph of a PMMA micro-wall part injection molded at an inordinately high mold temperature of $160{ }^{\circ} \mathrm{C}$ (Fig.14) clearly shows unsought molding with many voids caused by gas generation. The gas likely originates from the polymer material due to the high temperatures. 
The possibility of air presence in the cavity is not likely because of the use of vacuum evacuation and complete drying of the polymer before injection molding.

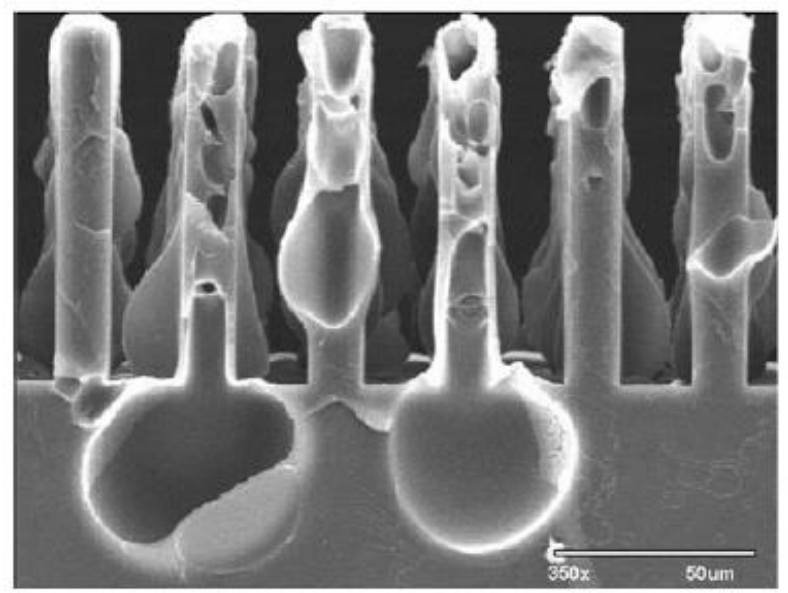

Fig.14. The effect of excessively high mold temperature on the PMMA micro-walls ${ }^{19}$

As a consequence, there are two overall issues which challenge the replication quality of injection molded micro-nanostructures. The first, which takes place in the processing stage, is incomplete filling of high aspect ratio micro-nanostructures by molten polymer. It is believed that the solution is increasing the mold temperature higher than polymer $T_{g}$ in order to decrease polymer viscosity adequately ${ }^{3}$. The other issue is attributed to interfacial adhesion between the polymer and the mold substrate. This challenge will be discussed further in the demolding section of this review.

\section{Variotherm Heating System}

The formation of a premature solid layer is the great challenge to micro-nanostructure injection molding systems. This solid layer restrains the polymer melt from completely filling the high aspect ratio micro-nanostructures. Increasing the mold temperature offers a possible solution, however, this also unfortunately lengthens cycle time. Therefore, a preferred solution is to have a cold mold during the cooling stage and a hot mold during the injection stage. This is the basis of the so-called "variotherm" system. Fig. 15 compares mold temperature between classical and variotherm processes ${ }^{57}$. The variotherm process employs numerous heating methods including heating by various types of heat transfer phenomena e.g. convection, radiation, conduction, and induction $^{92}$. 


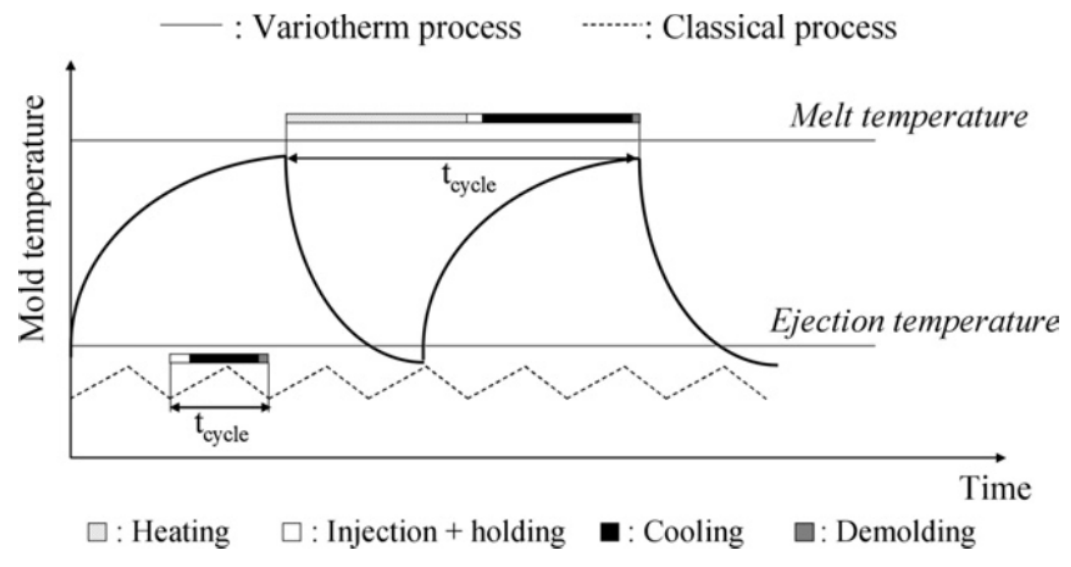

Fig. 15. Mold temperature in classical and variotherm processes ${ }^{57}$

The advantages of using this system include improving component quality, processing control and feature replication, increasing the polymer flow path and fluidity of the polymer, decreasing residual stresses, molecular orientation and flow resistance, and eliminating the formation of weld lines, short shots and material degradation ${ }^{57,92-94}$.

As shown in Fig.16, four samples were produced using variotherm system under two different warm circuit temperatures (see the reference ${ }^{92}$ ). The results are all highly satisfactory; there is no image of the samples produced without the variotherm system, however thus preventing a direct comparison.

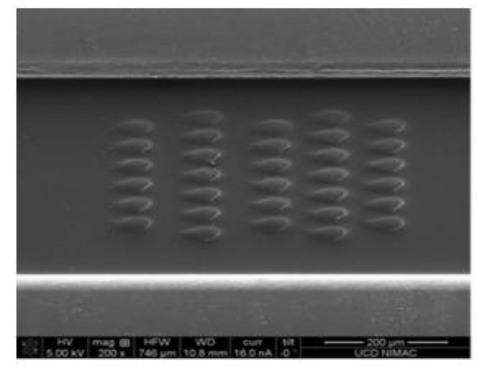

(a)

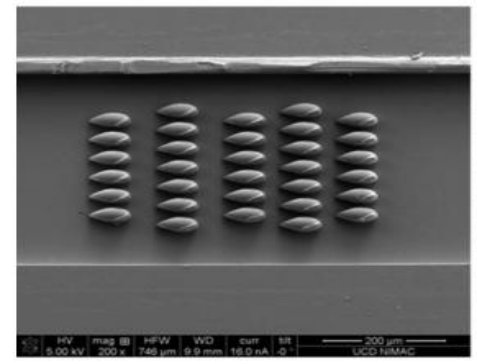

(c)

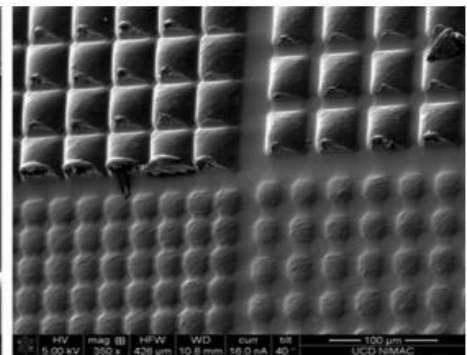

(b)

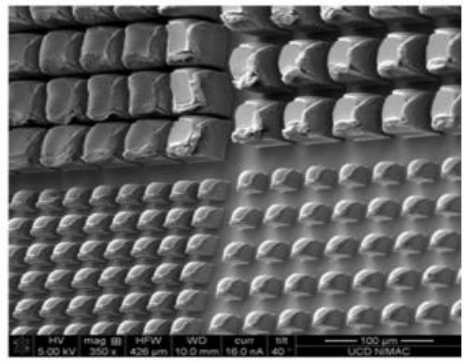

(d)

Fig.16. The SEM images of samples produced using variotherm system with various circuit temperatures: $130{ }^{\circ} \mathrm{C}(a$ and $b)$, 150 ${ }^{\circ} \mathrm{C}(\mathrm{c}$ and $d)$ and different patterns: water droplet shape $(a$ and $c)$ and square pillar features $(b \text { and } d)^{92}$. 
As a conclusion, variotherm processes can compensate for an increased cycle time induced by a traditional heating system while eliminating the emplacement of an unfavorable solid film layer; however this technique, due to the frequent and fast heating and cooling cycles, decreases the mold lifetime and also demands expensive equipment ${ }^{4,57}$.

\subsection{Melt Temperature}

In general, the effect of melt temperature on the replication quality is similar to the effects of mold temperature. An increase in the melt temperature decreases the viscosity of thermoplastic polymers, making it easier to fill the micro-nanostructures ${ }^{53}$. Therefore, high melt temperatures are advantageous for achieving a high quality micro-nanostructure. As such, many studies show high melt temperatures lead to an improved replication quality due to a decrease in the viscosity of the polymer ${ }^{53,95}$. In fact, high melt temperatures in the region of the micro-nanostructures provide a means for the holding pressure to force molten materials into the structures ${ }^{3}$. The increase of the melt temperature limits the increase of the "skin layer" thickness ${ }^{73}$. The highest possible barrel temperature, which is considered as the melt temperature, is needed to keep the materials in their flow state so that they can efficiently fill the structures of the cavity surface ${ }^{18}$.

The interaction of the melt and mold temperatures may be crucial for the injection molding of micro-nanostructures. An increase in the mold temperature and different melt temperatures showed varied replication qualities. An increase in the mold temperature has a positive role when the melt temperature is low and has a negative effect when the melt temperature is high. Consequently, to make a surface having a large contact angle, the best processing conditions are low melt and high mold temperatures ${ }^{38}$.

In the case of rubber injection molding, the role of melt temperature is completely different. Crosslinking rates increase with temperature, so in the rubber injection process, it is necessary to keep the temperature of raw material (uncured rubber) as low as possible before filling the cavity to prevent undesirable crosslinking. When the rubber material fills the cavity, it is time to cure in situ to create final cured rubber. If the mold temperature is high or the temperature of raw rubber is greater than the temperature in which the crosslinking bonds start to be created, an undesirable rubber curing happens. Under such conditions, i.e. uncontrolled curing before filling of the cavity, it is totally impossible for the rubber material to fill the micro-nanostructures. Hopmann et al. ${ }^{38}$ studied the injection molding of Liquid Silicone Rubber (LSR). They found an internal pressure in the mold cavity when the melt temperature is low enough. In other words, low temperature results in high viscosity that leads to high stress. This generated pressure can be a practical force to facilitate the penetration of rubber into the structures. Maximum heights were achieved at lower melt temperatures.

However, a change in the melt temperature may also have no effect on the replication quality or a negligible gradual effect ${ }^{39}$. For example, a $10^{\circ} \mathrm{C}$ increase in the melt temperature does not significantly influence the achieved height ${ }^{41}$. It is also mentioned that the increase in the melt temperature can lead to an increase in the degradation rate of polymer during processing ${ }^{23}$.

\subsection{Holding Pressure}

The main driving force that pushes the polymer melt into the micro-nanostructures of the cavity surface is packing pressure. Indeed, molten polymer fills the micro-nanostructures in the packing 
stage $^{3,53}$. No matter how high the mold and melt temperatures are, a great pressure is needed to push the molten material into the structures. In addition to the role of holding pressure in pushing the polymer into the holes, it also could compensate for the trapped air pressure and polymer shrinkage ${ }^{28,53}$. It has been reported that pressure over $150 \mathrm{MPa}$ is an appropriate holding pressure for a complete filling of micro-nanostructures, however too many processes work with lower pressures ${ }^{53}$.

The effect of holding pressure is highly dependent on the distance of the structures from the injection gate. Closer to the injection gate, the pressure is higher. Moving away from the gate, the pressure rapidly decreases because of the frictional shear forces due to the flow resistance. In many respects, the pressure at the farthest distances from the gate determines the replication quality of the final product ${ }^{18}$.

There is a remarkable interaction between injection velocity and holding pressure, as shown in Fig.17. The variation in the holding pressure significantly influences the feature height for high values of injection velocity. The results showed a $50 \%$ increase in the average height of the features with increasing pressure. However, the high values of injection velocity lead to a nonuniform pressure distribution that increases the standard deviation of the height values ${ }^{86}$.

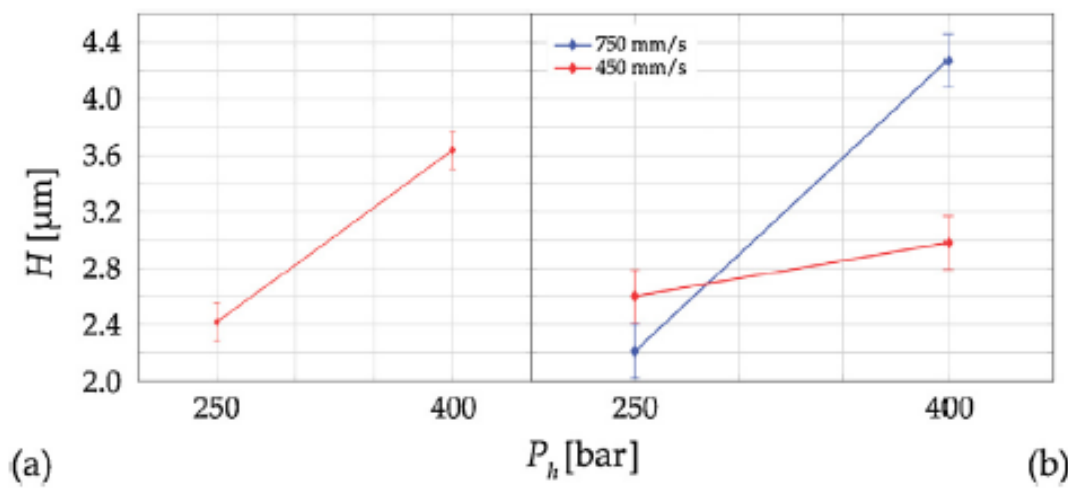

Fig.17. (a) Main effect of holding pressure and (b) interaction between holding pressure and injection velocity on the feature height ${ }^{86}$

A better replication for the surfaces with the micro-nanofeatures is thus obtained by a lower injection velocity and a higher holding pressure. This is shown in Fig.18 where max $\left(\mathrm{H}-\sigma_{\mathrm{h}}\right)(\mathrm{H}$ represents the average and $\sigma_{\mathrm{h}}$ represents the standard deviation of the height values) indicates the most desirable replication quality occurs at lowest injection velocities and highest holding pressures. 


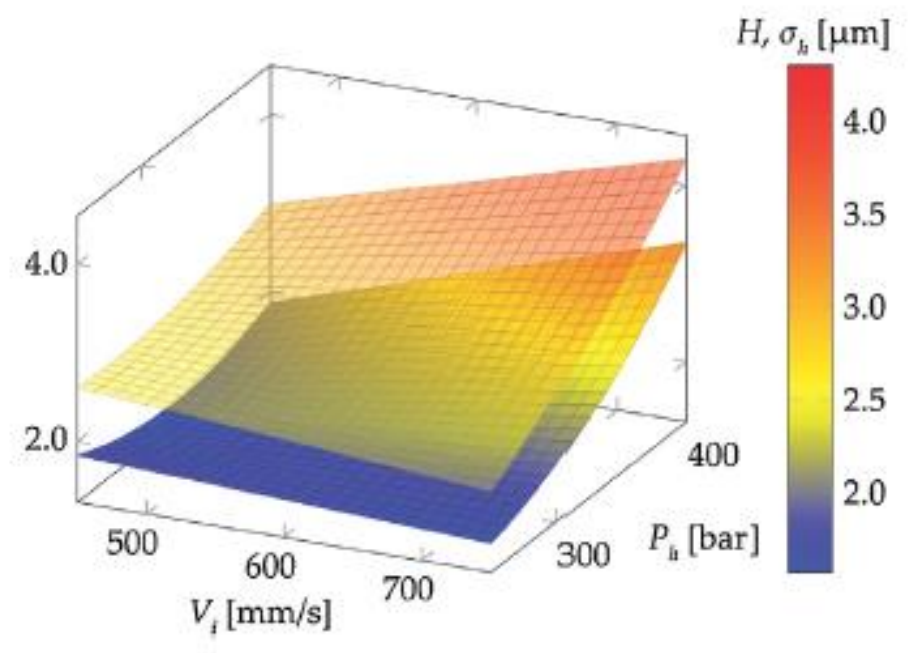

Fig.18. Response surfaces for the average (higher surface) and the standard deviation (lower surface) of the height values ${ }^{86}$

Fig.19 shows the influence of various holding pressures on the transcription ratio. However, it should be noted that at a particular injection rate, the effect of mold temperature is greater than that of holding pressure ${ }^{89}$.

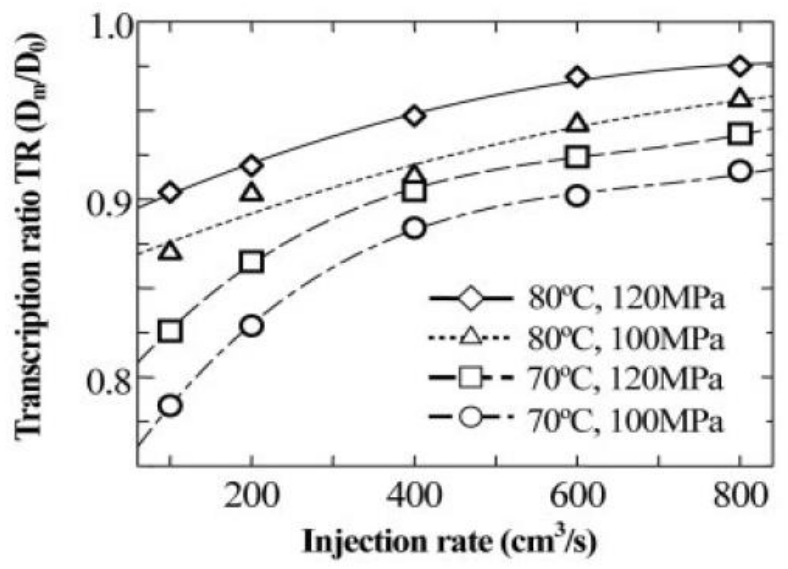

Fig.19. Transcription ratio (ratio of depths of $V$-grooves both in the molded samples and the stamper) versus injection rate with various mold temperatures and holding pressures 89

In general, the filling time of the cavity is much more than the solidifying time of the injected material in the vicinity of the mold surface. Accordingly, the formed skin layer between polymer bulk and the cavity surface counteracts the influence of packing pressure ${ }^{18}$. Indeed, holding the clamping force is useful as long as the molten polymer is in the flow state. Once the skin layer is formed, holding the pressure has no effect on the replication quality. Therefore, in majority of studies holding time is not considered as a crucial factor in the injection molding of micronanostructured surfaces ${ }^{20,86}$. 


\subsection{Injection Velocity}

Injection velocity is one of the most important yet one of the most debated processing parameters. Injection velocity can enhance the melt flow in the cavity and thereupon affects the features replication, especially at the locations away from the injection gate ${ }^{18,95}$. In general, a higher injection velocity has two positive effects that improve the replication quality $4,47,52,57,96-$ ${ }^{97}$ : i) a reduced viscosity as according to polymer rheology, a higher injection velocity means higher shear rates and shear stress. Consequently, the viscosity of polymer melt decreases with an increase of injection velocity, and ii) a reduced cooling rate during the filling step.

With an increase in injection velocity, the polymer melt has less time to cool down. In other words, as injection velocity increases, the contact time between the material and the cavity cold surface decreases. It prevents skin layer formation before the molten polymer fills the structures. Moreover, the undesirable short shots are also limited under these conditions.

This is particularly important in the case of rubber injection molding. In rubber injection molding, the mold temperature is higher than the melt temperature, so the polymer material in the filling stage is in contact with a hot cavity rather than a cold one. When the rubber compound is in contact with the hot cavity surface, crosslinks form more often and more quickly. This cured skin prevents the filling of micro-nanostructures by other polymer melts and the result is poor replication quality. Premature scorching should be necessarily avoided to obtain an acceptable filling. It has been claimed that a high injection velocity is the only factor that ensures a satisfactory filling in rubber injection molding as longer filling time inevitably leads to premature scorching ${ }^{38}$. When the injection velocity is high enough, the induced internal pressure in the cavity forces the molten material into the microfeatures at a desired level.

On the other hand, a dissipative heating of material due to a high injection velocity can occur ${ }^{38}$. This induced heating which results from the induced shearing by the high velocity of the polymer material, can generate a high melt temperature. This rise in melt temperature could lead to some undesirable consequences in the case of rubber injection molding. Therefore, an appropriate injection velocity during the rubber injection process has a considerable influence on the final results.

Song et al. ${ }^{73}$ verified the influence of injection velocity on the replication quality. Working on the replication of large scale micropillar arrays having different diameters, they used three injection velocities: low, intermediate, and high. The mean height of the micropillars decreased when injection velocity is low. In the case of intermediate injection velocity, due to the similarity of the melt temperature at both the beginning and the end of the filling process, the acquired aspect ratio was constant and parallel. Finally, when injection velocity was higher, there was no difference in the height of the micropillars in the onward and the opposite directions, meaning micropillars height remained constant. As such, injection velocity and the mold temperature were selected to be as high as possible.

Undoubtedly, complete filling is not related to an increase in the injection velocity. If a high injection velocity cannot ensure a complete filling, it can at least lead to a more uniform and homogeneous molding of the structures, an aspect that has a great effect on the water contact angle of the surface ${ }^{38}$. 
According to Yokoi et al. ${ }^{89}$ the most practical way to avoid the formation of solidified thin layer is using high injection velocities. They used ultra-high-speed injection molding (UHSIM) to process at a high injection rate. In comparison with conventional injection molding process having an injection rate of about $50-100 \mathrm{~mm} / \mathrm{s}$, UHSIM can inject the polymer into the mold at a rate of $1000-2000 \mathrm{~mm} / \mathrm{s}^{89}$.

Contradictory effects of increasing the injection velocity were also observed. Higher injection velocities can lead to the improved replication by increasing shear stress and decreasing polymer viscosity. This phenomenon is called shear thinning. On the other hand, this generated shear stress may cause an induced elastic stress residual in the polymeric parts after demolding. As a solution, a high mold temperature will decrease viscosity without inducing shear thinning and a subsequent elastic stress relaxation in the final part ${ }^{41,98}$.

A non-monotonic behavior of the replication depth with increasing injection velocity is presented in Fig. 20. With the increase in the injection flow rate up to $4 \mathrm{~cm}^{3} / \mathrm{s}$, replication depth increases, yet above these values, replication depth is reduced ${ }^{5}$.

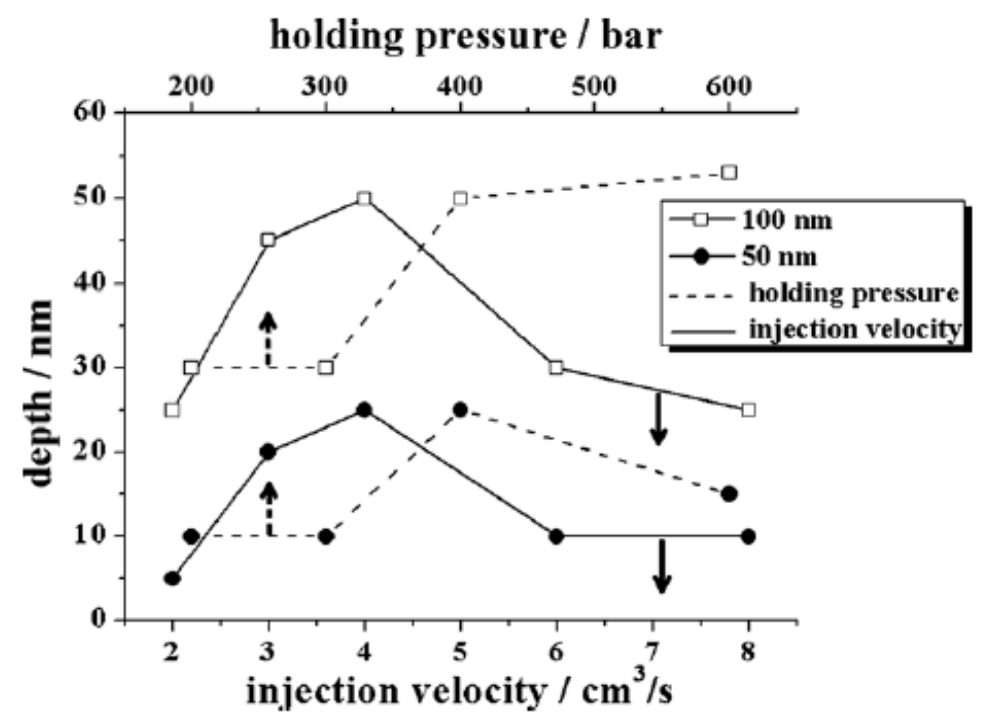

Fig.20. Replication depth of 50 and $100 \mathrm{~nm}$ wide holes as a function of injection velocity (lower axis) or holding pressure (upper axis $)^{5}$

To obtain high aspect ratio and desirable replication quality, injection velocity has to be set at a minimum level ${ }^{86}$. An increase in the injection velocity causes a marked increase in the trapped air pressure between the polymer melt and the cavity surface ${ }^{14}$. The trapped air leads to a poor surface quality in the final product. An active vacuum-based method is suggested to solve this problem and guarantee the complete air evacuation ${ }^{19,99}$.

Although high injection velocity improved the filling of micro-nanostructures, poor surface quality and edge definition in demolded parts have also been observed ${ }^{95}$. An enhanced feature sizes has been noted in the cases of using low injection velocities ${ }^{41}$. The replication of all feature 
sizes except smallest ones $(100 \mathrm{~nm})$ were improved as the injection velocity decreased. This observation could be attributed to the similarity between the hot embossing process and the injection molding process at zero injection velocity. However, this claim would need to be verified.

As a conclusion, one of the most questionable and yet effective parameters is injection velocity. Therefore, the selection of injection velocity highly depends on other processing conditions such as type of polymer, mold and melt temperature, and holding pressure.

\subsection{Part Thickness}

The thickness for the molded part (also known as substrate) is also a strongly debated subject in terms of the role of processing parameters in the injection molding process of micro-nanofeature surfaces. Some have argued that with a decrease in the part thickness comes an improvement of the filling capability; others state that a reduced part thickness markedly deteriorates replication quality.

The geometry of the substrate affects the pressure profile which in turn affects the filling capability of the molten polymer. Indeed, the thickness of the substrate is much larger than the microfeatures. Therefore, it could easily change the pressure profile and, in doing so, affect replication quality ${ }^{18}$. In fact, thicker substrates lead to a deterioration in replication quality as it limits increases in cavity pressure during the molding process. In contrast, thinner substrates favor an increase of in-cavity pressure that tends to increase the height of fillings.

Some other results complicate the role of part thickness. As wall thickness decreases, the injection molding of microfeatures becomes more difficult. Increased substrate thickness leads to a uniform distribution of cavity pressure in the holding stage. Therefore, to improve replication quality, a greater wall thickness becomes essential ${ }^{19,86}$. A thicker substrate is recommended to increase the filling rate and prevent the creation of a solid thin layer ${ }^{100}$.

Clearly, as the thickness of the molded part increases, given a constant injection rate, the cavity filling time increases ${ }^{18}$. Sum told, a precise and comprehensive design for a part must consider thickness to produce a favorable result.

\subsection{Filling distance}

The distance of micro-nanofeatures from the injection gate influences the replication quality. In general, as the structures are closer to the injection gate, replication quality is better. Furthermore, processing parameters also play an important role with respect to filling distance. It is believed that due to the higher holding pressure closer to the injection gate, the replication quality is better in these regions of the mold. As shown in Fig.21 and Fig.22, filling height quickly decreases as the distance from the gate increases ${ }^{86}$. 

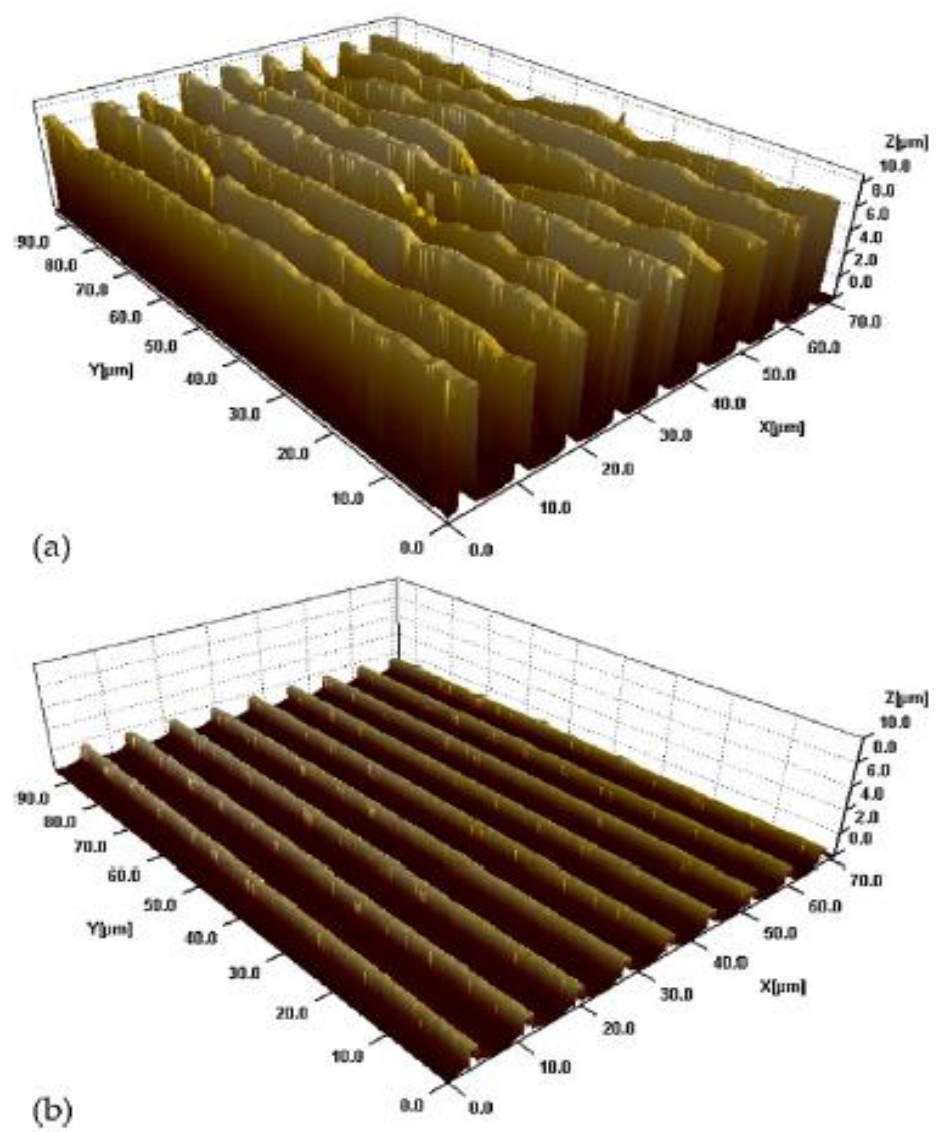

Fig.21. Replicated microfeatures located at (a) $1.5 \mathrm{~mm}$ and (b) $35 \mathrm{~mm}$ from the gate ${ }^{86}$

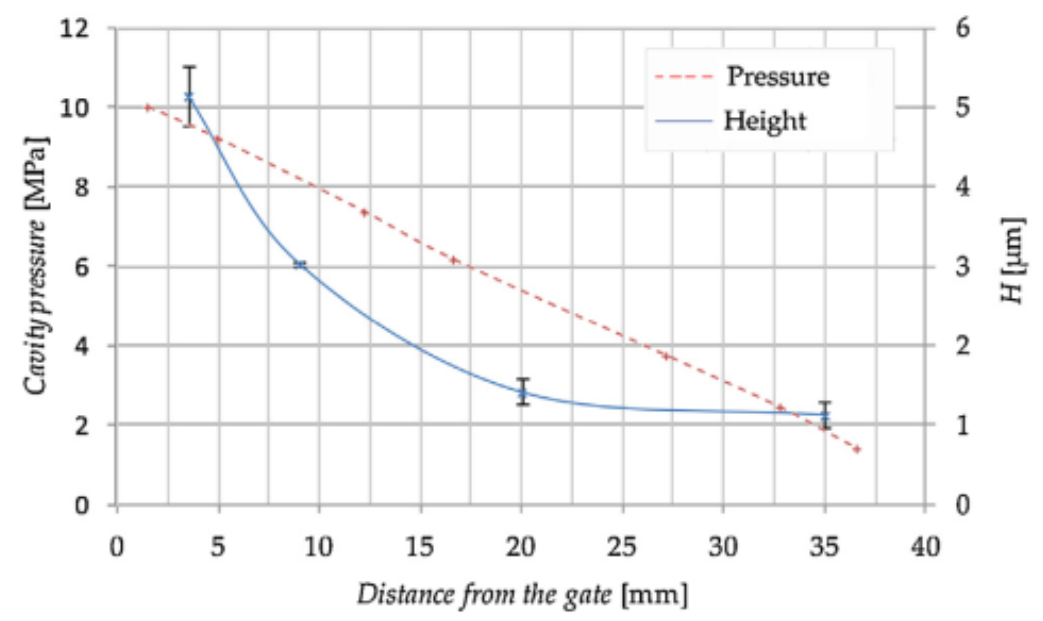

Fig.22. Comparison between cavity pressure and microfeatures height as a function of the distance from the gate ${ }^{86}$

Su et al. ${ }^{42}$ studied the influence of radial location on the replicated aspect ratio. As shown in Fig.23, the obtained aspect ratio is not uniform between different cavity sizes. The replicated 
microstructures near the gate have higher depth-to-opening ratios (h/d) due to the higher local temperature and pressure. However, as radial location increases, the aspect ratio decreases, except in the areas close to the edge of the mold. Along the edge, the generated back pressure increases. This means that the pressure applied to the molten polymer increases as the materials are facing to a closed area. The depth-to-opening ratio increases again even more than that at the central area.

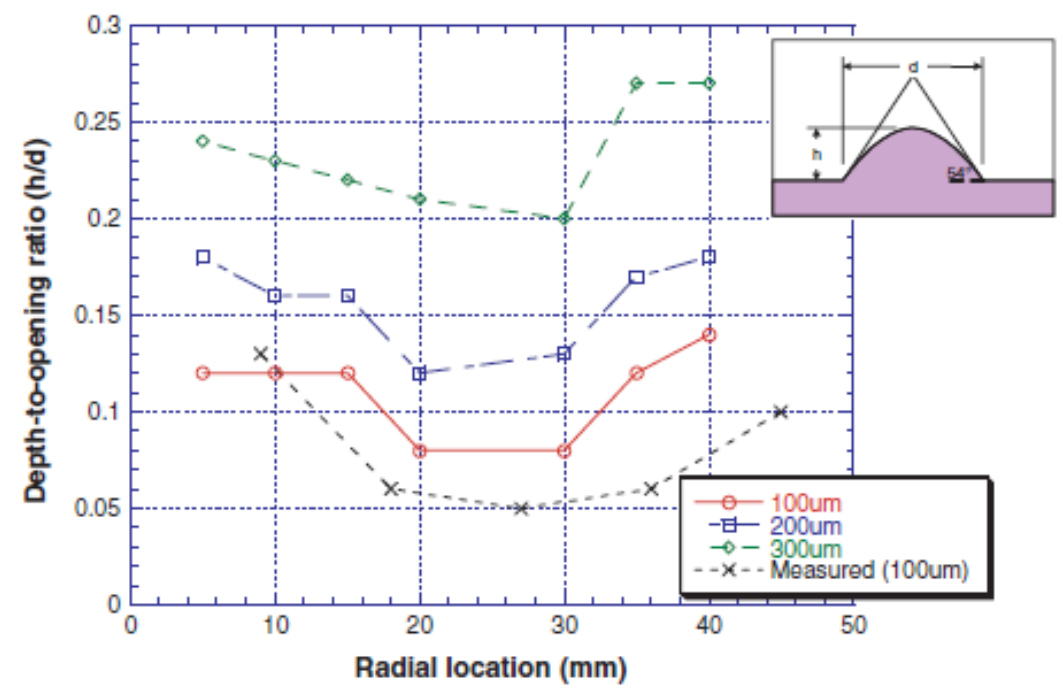

Fig.23. Simulation results of molding quality versus radial location with various cavity sizes ${ }^{42}$

Other studies, however, have shown an opposite, positive relationship between replication quality and the distance from the gate ${ }^{42,95,101}$.

Although the distance of the structures from the gate is believed to be an important, albeit debatable, parameter affecting the replication quality, distance between micro-nanostructures has no significant effect on the filling efficiency ${ }^{95}$.

\subsection{Vacuum mold venting (air evacuation)}

The presence of air trapped in the mold cavity creates a major problem during molding and results in voids and bubbles in the bulk or surface of the final assembled part, incomplete filling — known as short shot, poor appearance, surface combustion-known as the diesel effect, burn marks, and in some cases permanent damage to the mold ${ }^{14,89,102}$.

The presence of air in the mold is inevitable. Therefore, it is critical to remove the air from the mold cavity. Since the features are susceptible to any dimensional change, these problems are much more crucial for the injection molding of nanostructures ${ }^{89}$. Numerous solutions for the evacuation of this trapped air have been proposed ${ }^{16,19,52,103-104}$.

Two different methods are used to avoid obtaining voids in the cavity: i) conventional venting using particular vents created on the mold walls to let the trapped air escape, and ii) air evacuation using pumps to thoroughly remove air. In standard injection molding, air vents provide a solution. However, due to the small sizes of the micro injection molding parts and 
molds; this conventional approach is not a feasible solution. Conventional venting leads to unfavorable structural changes in the micro molded materials ${ }^{42}$. However, the combination of a vacuum pump and small holes is used to evacuate the trapped air and prevent poor replication of the microstructures ${ }^{104}$.

Yokoi et al. ${ }^{89}$ used the transcription ratio (TR) to illustrate the effect of different processing parameters on the quality of filling with or without vacuum pumping. TR is defined as the ratio of the depths of V-grooves within the molded sample to those of the stamper. The achieved aspect ratio was greater in the case of using vacuum pumping. In contrast, a study of molding with air evacuation produced a $16 \%$ reduction in the average height of the microstructures $(\mathrm{H})$ (Fig.24). The main parameter increasing the aspect ratio is mold temperature. The existence of the vacuum mold venting evacuates warm air within the cavity, leading to decreased the mold temperature and, as a consequence, there is a deterioration of replication quality ${ }^{14,86}$. This adverse effect is especially dominant in the case of molding polymers that are sensitive to temperature change such as polystyrene ${ }^{86}$. Therefore, it has been claimed that to obtain a surface with desirable average height of the micro-nanostructures, use of air evacuation should be avoided.

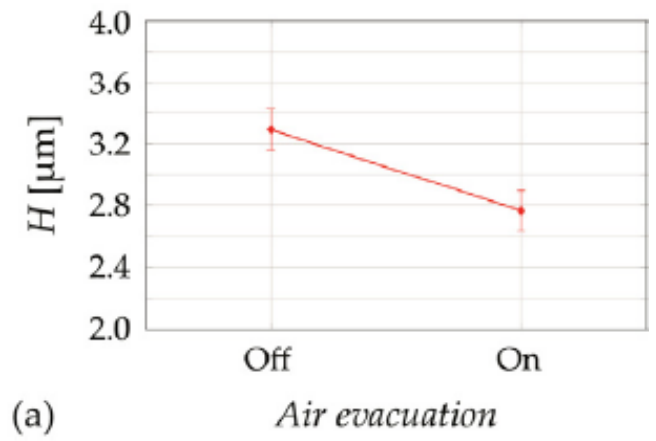

Fig.24. Main effect of air evacuation on the feature height ${ }^{86}$

\subsection{Injection pressure}

Finally, injection pressure is attributed to the pressure by which the materials are injected into the mold cavity. Evidently, injection pressure affects the flowability of polymer melt and the replication quality ${ }^{105-106}$. The replicated aspect ratio increases with a greater main injection pressure. This tendency to increase is not monotonic. Fig. 25 shows that the rate of increase for the achievable aspect ratio tends to flatten as the injection pressure increases ${ }^{19}$. Some researchers consider injection pressure as the secondary factor affecting the filling capacity of the micro-nanostructures ${ }^{107}$. 


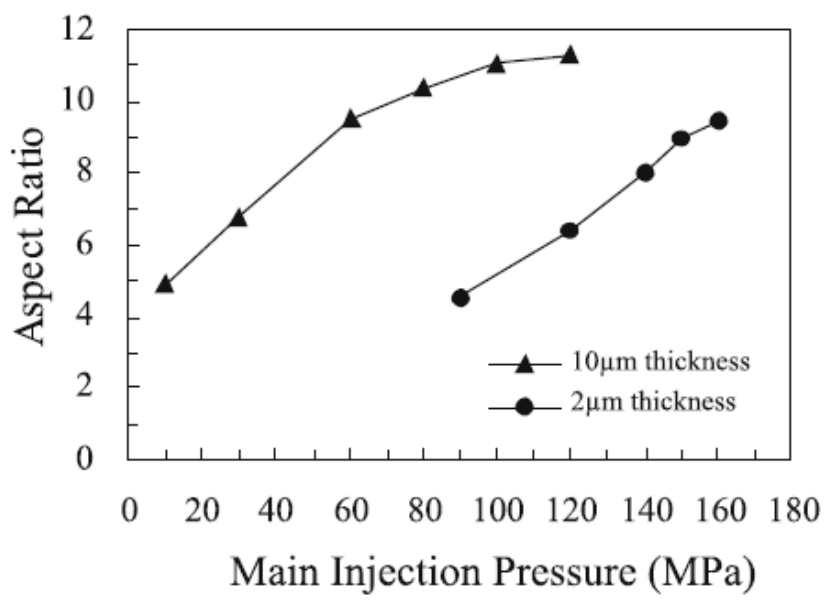

Fig.25. Effects of main injection pressure on the obtained aspect ratio of the micro-walls with two different thicknesses ${ }^{19}$

In addition to these processing parameters, other, less studied parameters that may affect the replication of the micro-nanostructures include maximum ejection temperature ${ }^{104}$, metering size, main injection time ${ }^{19}$, type of runners (hot or cold) ${ }^{57}$, and movement of the injection plunger in order to control the holding pressure ${ }^{108}$. These parameters directly or indirectly affect the main aforementioned parameters.

\section{Demolding}

Both processing parameters and demolding conditions affect the final heights of the replicated micro-nanopatterns. In addition to a complete mold filling, high quality replication requires a flawless demolding where all micro-nanostructures withstand demolding forces. Inappropriate design of demolding forces used to remove the manufactured piece from the cavity can lead to irreparable structural deformation or even failure of the molded features on the polymer surface, and also can affect the lifetime of the mold ${ }^{3,109-110}$.

Accurate design is needed considering many factors from polymer selection and mold conditions to processing parameters and part design ${ }^{111}$. Applied forces to the polymer surface, difference in the thermal conduction coefficient between the polymer material and mold metal, generated forces due the shrinkage of the polymer during the solidification stage, process parameters, cavity shape and material, molding material and geometry, features shape and aspect ratio-to be name but a few ${ }^{109}$.

There are two different main demolding methods. Either one or both of these methods can be used to release the final product from the mold. The first uses demolding chemical surface agents named antistiction coatings. The second is a mechanical ejector such as pins, blades, rings, sleeves, and stripper blades ${ }^{20,109,112}$. Both methods have advantages and disadvantages. Use of chemical demolding agents is restricted in the medical or some microfluidic applications due to probable harmful effects on human health ${ }^{57}$. Mechanical ejectors can lead to permanent deformation especially when the part geometry is complicated or the distribution of the ejector pins is not appropriate ${ }^{28,109}$. Therefore, a proper design of produced tool and the location of ejector pins is necessary to avoid damage and failure to the polymeric parts ${ }^{20}$. To circumvent the 
disadvantages of both chemical and mechanical demolding approaches, novel methods such as ultrasonic vibration ${ }^{57}$ and reduction the surface roughness to decrease the coefficient of friction of the mold surface ${ }^{113}$ require further investigation.

In general, demolding is comprised of two different forces (Fig.26): adhesion and friction. Adhesion is the force at the intersection of the microfeature bottom and the top surface of polymer material. Friction is the force produced by the movement of the molded polymer inside the feature along the walls of the microfeature. The force acts in the opposite direction to the ejection movement ${ }^{109}$.

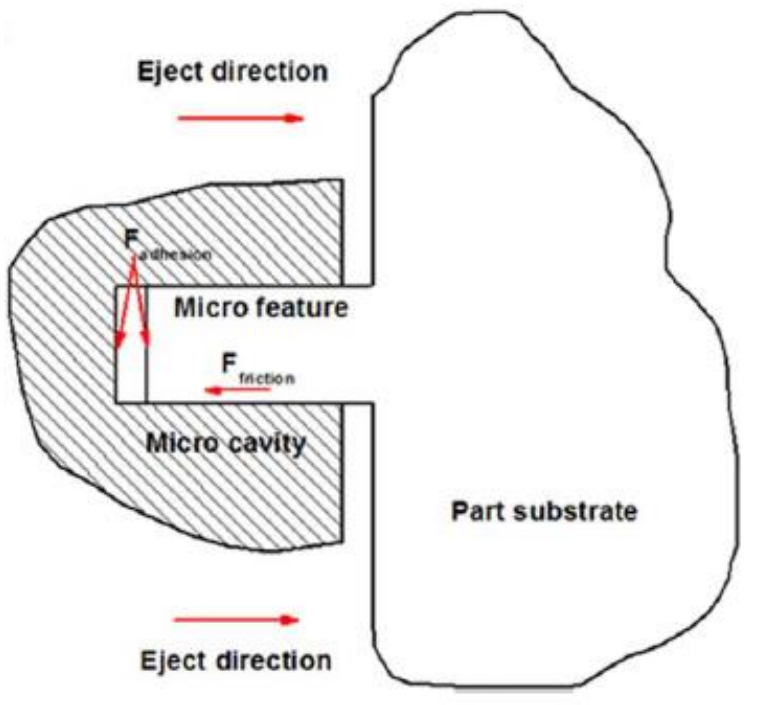

Fig.26. Illustration of the demolding forces 109

The effects of the processing parameters on the demolding forces are frequently reported. The increase in both holding pressure and mold temperature leads to a decrease in the demolding force ${ }^{114-115}$. Increased holding pressure can reduce the shrinkage and consequently decrease demolding forces. Evidently, longer cooling times lead to higher demolding forces ${ }^{109}$. Moreover, melt temperature and injection pressure also influence demolding forces ${ }^{109,116}$.

Fig.27 shows the results of the "Design of Experiments using Taguchi method" on the effects of the processing parameters on the demolding forces. The mold temperature is the dominant parameter determining demolding forces ${ }^{109,117}$. Melt temperature has a moderate effect with packing pressure and time having negligible effects ${ }^{109}$. 


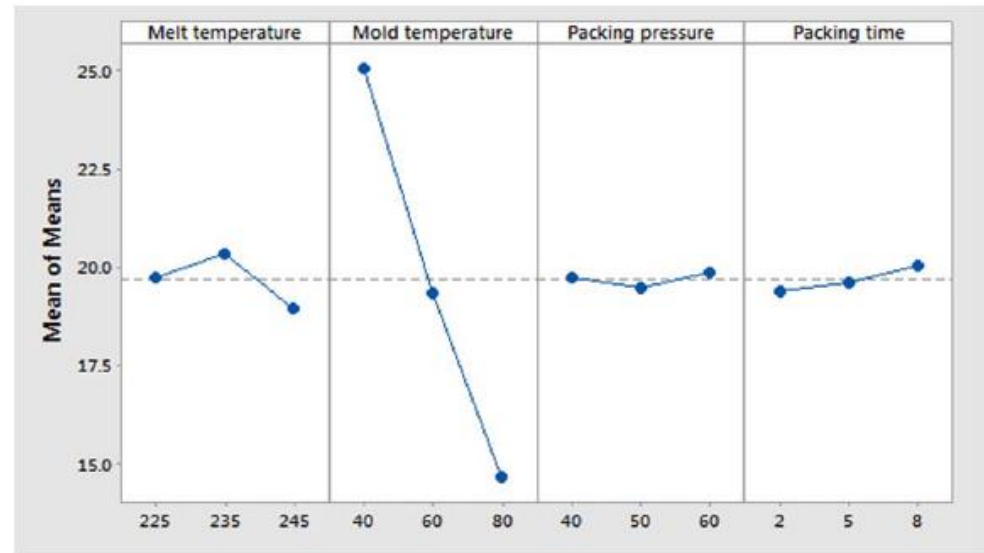

Fig.27. The main effects plot of the data ${ }^{109}$

Moreover, part thickness affects the demolding forces (Fig.28) where as thickness increases, the demolding force decreases but not monotonically.

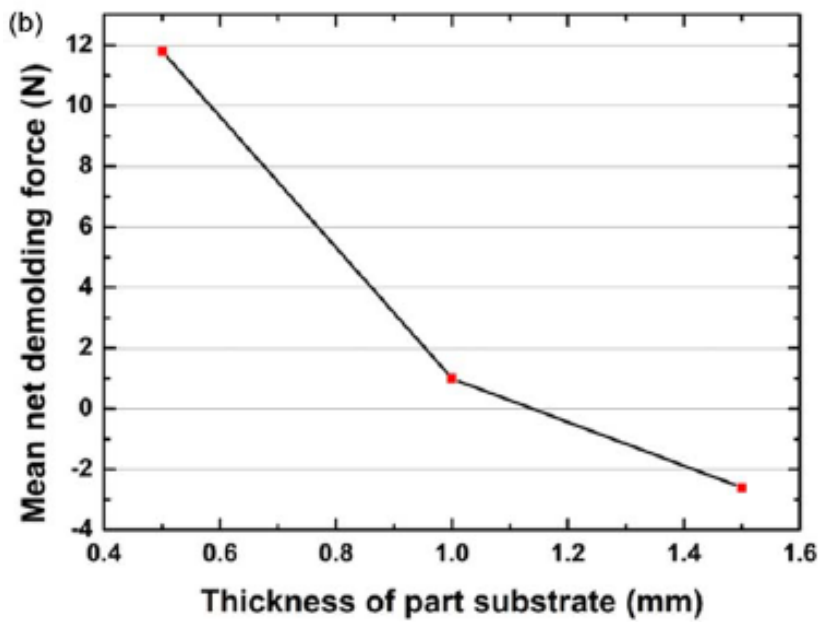

Fig.28. The influence of the part thickness on the demolding forces ${ }^{109}$

\section{Antistiction Coating}

Although demolding or release agents are suitable for larger components, they could affect the replication dimensions in case of micro replication processes ${ }^{110}$. The use of antistiction coatings can improve the quality of the molded surface and the uniformity of the nanostructures while preserving surfaces without any microcracks and polymer residues ${ }^{3}$. The low surface energy coatings are commonly used as the antistiction coatings using self-assembled monolayer method 5. In general, fluorocarbon or hydrocarbon based coating materials such as fluorocarbonsilanes ${ }^{118-121}$, fluorocarbon phosphoric acid ${ }^{3}$, fluorocarbons ${ }^{54}$ or alkanethiols ${ }^{3}$ are the most common antistiction coatings. Specifically, perfluorodecyltrichlorosilane (FDTS) has been used as an effective antistiction coating.

Equation $3^{3}$ can be used to calculate the maximum tensile stress $\sigma_{\text {pillar }}$ on a cylindrical pillar. The friction force $F_{\text {fric }}$ is calculated based on shear stress $\sigma_{\text {macro }}$ which is required for 
detachment of polymeric parts from the mold surface. The cross-sectional area $A_{\text {pillar }}$ of a cylindrical pillar can be calculated by its diameter $w$ and height $h$ :

$$
\sigma_{\text {pillar }}=F_{\text {fric }} / A_{\text {pillar }}=4 \sigma_{\text {macro }} h / w
$$

Eq. 3

Based on Eq. 3, the demolding forces for a nanopillar of diameter $40 \mathrm{~nm}$ and height $110 \mathrm{~nm}$ can be calculated with and without mold coating. The maximum tensile stress of demolding from a FDTS coated mold would be $110 \mathrm{MPa}$, while in the case of unmodified mold, the needed demolding stress would be more than $550 \mathrm{MPa}^{3}$.

Moreover, as shown in Fig.29 andFig.30, it has been claimed that the average pillar height of the samples produced by FDTS coated inserts was higher than that without antistiction coating. These results were not dependent on mold temperature ${ }^{3}$.
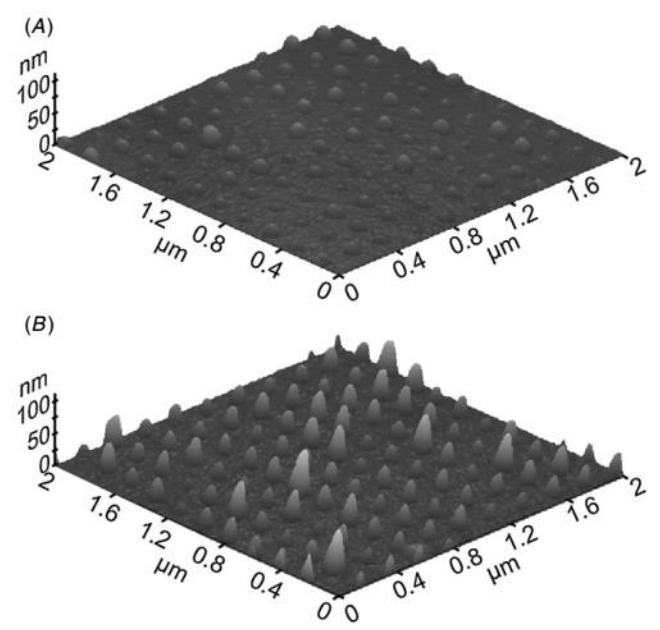

Fig.29. AFM micrographs of injection molded replicas on a native nickel mold insert at a mold temperature of (A) $60^{\circ} \mathrm{C}$ and $(B)$ $90{ }^{\circ} \mathrm{C}^{3}$
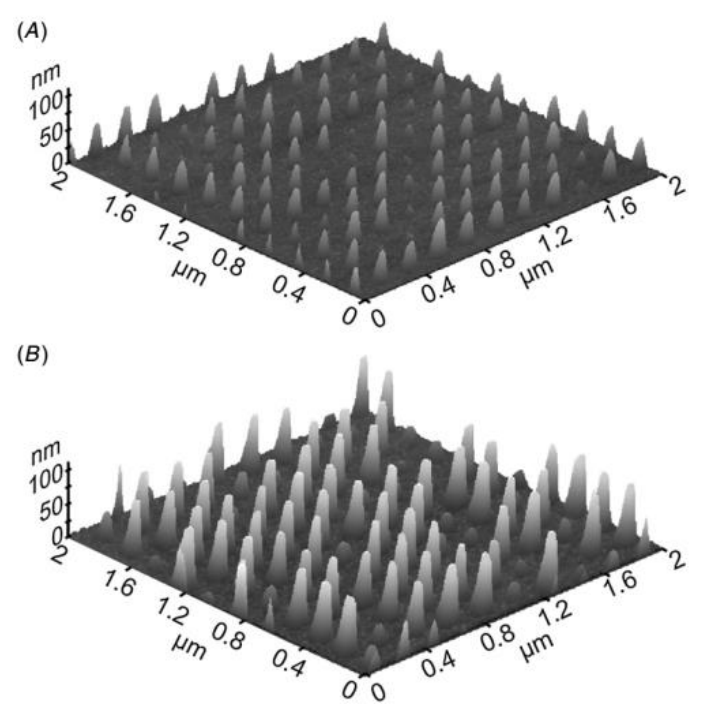

Fig.30. AFM micrographs of injection molded replicas on a fluorocarbonsilane modified nickel mold insert at a mold temperature of (A) $60^{\circ} \mathrm{C}$ and $(B) 90^{\circ} \mathrm{C}^{3}$ 
To achieve a desirable demolding, tensile forces must be able to overcome the aggregate polymer/mold adhesive and friction forces. Indeed, this demolding stress should not exceed the ultimate tensile strength of polymer bulk otherwise the molded polymeric structures would fracture. Fig.31 shows this phenomenon and also the positive effect of FDTS coating on demolding ${ }^{3}$.

(A)

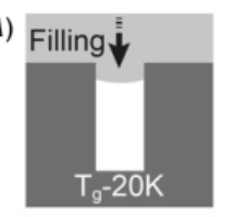

(B)

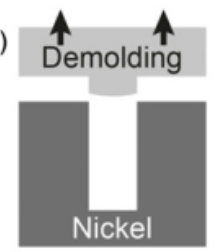

$(C)$

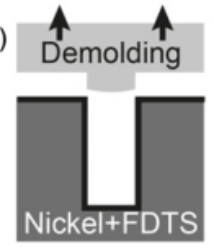

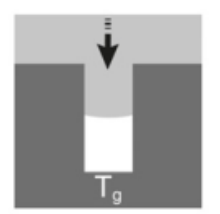
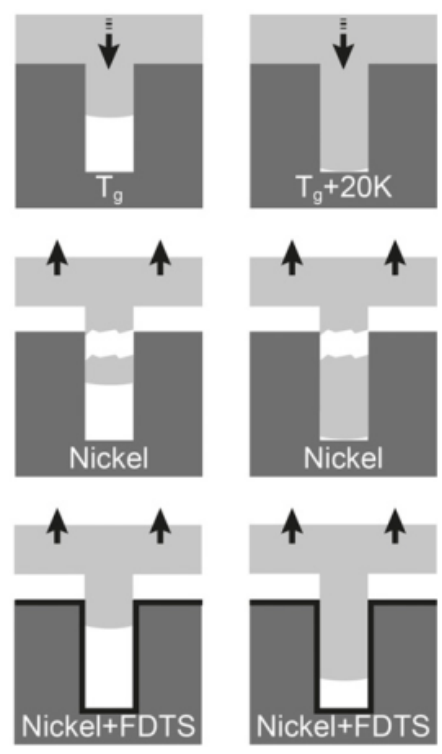

Fig.31. Schematic of the filling and demolding processes: (A) Filling of mold nanostructures with different temperatures (below $T_{g}$, at $T_{g}$, and above $T_{g}$ ) by polymer melt. $(B)$ Demolding at a mold temperature far below $T_{g}$ leading to fracture of structures. (C) FDTS coated nickel molds at a mold temperature far below $T_{g}$ leading to a proper demolding ${ }^{3}$

Although fluorosilanes are a group of known antistiction coatings in the existing literature, some modification may be required ${ }^{1,122}$. It is almost impossible to find a coating suitable for all molding processes; however, some techniques such as water contact angle measurement could be a useful way to efficiently select a proper antistiction coating. An expanded presentation of surface coatings used to improve replication quality is provided in the literature ${ }^{1}$.

The antistiction coating consistency is considered as a critical factor for obtaining favorable demolding results. As it is illustrated in Error! Reference source not found., a stretching effect was observed in the case of inconsistent coating. The increase in the friction between mold surface at the bottom of the structure and the polymer material caused this stretching phenomenon. However, a consistent antistiction layer guarantees ideal demolding of the material without any stretching effect ${ }^{1}$. The stretching effect during demolding has also been observed when the polymer melt neighboring the mold surface remained above $\mathrm{T}_{\mathrm{g}}{ }^{39}$.

Error! Reference source not found.shows an acquired aspect ratio of over 20:1 in the injection molding of PC nanopillars with $\mathrm{SiO}_{2}+\mathrm{TPFS}$ (Trichloro(1H,1H,2H,2H-perfluorooctyl) silane) coating ${ }^{1}$. 


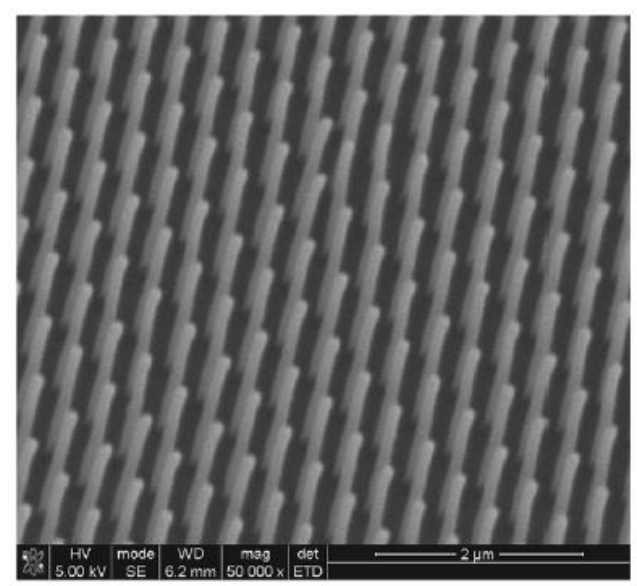

Fig.32. SEM image of PC nanopillars with aspect ratio 20:1 produced by injection molding with SiO2+TPFS coated inlays ${ }^{1}$

Stormonth-Darling et al. ${ }^{1}$ proposed the term of "success rate" to clarify the content of perfectly formed pillars. The success rate is defined as a ratio between the total number of perfectly formed features to the total number visible at the specified surface. In this definition, the broken or low height features generated by the improper filling are not considered as successful replicated pillars. A successful replication is achieved when there is no overlapping feature and the feature has adequate contrast to its neighbors. Three fluorinated coatings, TPFS-only, $\mathrm{SiO}_{2}+\mathrm{TPFS}$, and $\mathrm{Si}_{3} \mathrm{~N}_{4}+\mathrm{TPFS}$ demonstrated a greater stretching capability ${ }^{1}$. The success rate for the fluorinated coatings was generally above $80 \%$. On the other hand, the metal surfaces such as $\mathrm{Ni}$ and $\mathrm{Ti}$ were unsuccessful in fully filling the ultra-high aspect ratio structures while their success rate in terms of stretching capability was close to $100 \%{ }^{1}$.

Other antistiction coatings include a plasma-polymerized fluorocarbon-based coating that showed a significant improvement in replication quality due the use of a $10 \mathrm{~nm}$ fluorocarbon layer on a nickel mold surface ${ }^{5}$. This antistiction coating did not reduce the replication depth and the coating layer did not undergo any degradation even after hundreds of injection molding cycles 5 .

The effect of using antistiction coating has been studied on the injection molding of thermoplastic polyurethane (TPU) with silicon tooling ${ }^{98}$. TPFS was used as the antistiction coating material using a vapor self-assembled monolayer (VSAM) method. The depth ratio increased more than two fold at higher mold temperatures, while in the lower mold temperatures the increase of depth ratio is much less. In other words, the antistiction coating improves the effect of mold temperature.

Although there are many investigations which acknowledge the positive influence of antistiction coatings on the replication quality of micro-nanostructures ${ }^{41}$, there are some studies which consider a detrimental effect for the application of low surface energy coatings on the filling capability. For example, due to the insufficient wetting of the coating by the polymer melt, the antistiction coating limited the filling of nanostructures 5 . 
As a consequence, a negligible deformation during the demolding step is as important as a complete filling during the processing ${ }^{39}$. Indeed, a complete filling and a successful demolding can both guarantee the quality of final replication.

\section{Summary}

The demand for micro-nanofeatured parts is increasing rapidly due to the numerous applications in the different high-tech areas. Therefore, micro-nano injection systems are recognized as a promising industrial tool for rapid and precise fabrication to supply this demand. In this review, we discussed different aspects of injection molding of micro-nanostructured polymer surfaces across the three main steps of inserts (pre-processing), processing, and demolding (postprocessing).

The effects and opportunities of various insert materials, particularly the polymeric and hybrid inserts were assessed. Modification of an insert's surface must be accompanied by the appropriate processing conditions to produce the desired output. High quality replication of pieces includes favorable filling and demolding conditions obtained through a carefully selected set of processing parameters. Decisions regarding the exact specifications to use involve multiple aspects including the type of employed polymer, the size and shape of the mold, the size and shape of the structures. Mold temperature, injection velocity, and holding pressure are the most important parameters affecting the quality of the final product in injection molding processing.

It is crucial to find the best-matched condition to achieve the most favorable processing output as the effects from the main parameters are not consistent across variable processing conditions. Moreover, the capabilities of various demolding methods should be considered in decisionmaking and planning as a proper non-damaging demolding is required after a complete filling in the processing step to assure high quality replication.

\section{References}

1. Stormonth-Darling, J. M.; Pedersen, R. H.; How, C.; Gadegaard, N., Injection moulding of ultra high aspect ratio nanostructures using coated polymer tooling. Journal of Micromechanics and Microengineering 2014, 24 (7), 075019.

2. Moon, S.-d.; Lee, N.; Kang, S., Fabrication of a microlens array using micro-compression molding with an electroformed mold insert. Journal of Micromechanics and Microengineering 2002, 13 (1), 98 .

3. Matschuk, M.; Larsen, N. B., Injection molding of high aspect ratio sub-100 nm nanostructures. Journal of Micromechanics and Microengineering 2012, 23 (2), 025003.

4. Park, S.; Lee, W.; Moon, S.; Yoo, Y.; Cho, Y., Injection molding micro patterns with high aspect ratio using a polymeric flexible stamper. eXPRESS Polym. Lett 2011, 5 (11), 950-958.

5. Matschuk, M.; Bruus, H.; Larsen, N. B., Nanostructures for all-polymer microfluidic systems. Microelectronic Engineering 2010, 87 (5), 1379-1382.

6. Jafari, R.; Asadollahi, S.; Farzaneh, M., Applications of plasma technology in development of superhydrophobic surfaces. Plasma Chemistry and Plasma Processing 2013, 33 (1), 177-200.

7. Momen, G.; Farzaneh, M., Simple process to fabricate a superhydrophobic coating. Micro \& Nano Letters 2011, 6 (6), 405-407. 
8. Momen, G.; Farzaneh, M. In Superhydrophobic RTV silicone rubber coatings on anodized aluminium surfaces, Materials Science Forum, Trans Tech Publ: 2012; pp 2546-2551.

9. Jafari, R.; Farzaneh, M., Development a simple method to create the superhydrophobic composite coatings. Journal of Composite Materials 2013, 47 (25), 3125-3129.

10. Momen, G.; Farzaneh, M.; Jafari, R., Wettability behaviour of RTV silicone rubber coated on nanostructured aluminium surface. Applied Surface Science 2011, 257 (15), 6489-6493.

11. Momen, G.; Jafari, R.; Farzaneh, M., Ice repellency behaviour of superhydrophobic surfaces: Effects of atmospheric icing conditions and surface roughness. Applied Surface Science 2015, 349, 211218.

12. Hansen, H. N.; Hocken, R.; Tosello, G., Replication of micro and nano surface geometries. CIRP Annals-Manufacturing Technology 2011, 60 (2), 695-714.

13. Michaeli, W.; Spennemann, A.; Gärtner, R., New plastification concepts for micro injection moulding. Microsystem technologies 2002, 8 (1), 55-57.

14. Yang, C.; Yin, X.-H.; Cheng, G.-M., Microinjection molding of microsystem components: new aspects in improving performance. Journal of Micromechanics and Microengineering 2013, 23 (9), 093001.

15. Whiteside, B.; Martyn, M.; Coates, P.; Allan, P.; Hornsby, P.; Greenway, G., Micromoulding: process characteristics and product properties. Plastics, rubber and composites 2003, 32 (6), 231-239.

16. Ruprecht, R.; Gietzelt, T.; Müller, K.; Piotter, V.; Haußelt, J., Injection molding of

microstructured components from plastics, metals and ceramics. Microsystem Technologies 2002, 8 (4-5), 351-358.

17. Greener, J.; Wimberger-Friedl, R., Precision injection molding. Carl Hanser, Munich 2006.

18. Masato, D.; Sorgato, M.; Lucchetta, G., Analysis of the influence of part thickness on the replication of micro-structured surfaces by injection molding. Materials \& Design 2016, 95, 219-224.

19. Liou, A.-C.; Chen, R.-H., Injection molding of polymer micro-and sub-micron structures with high-aspect ratios. The International Journal of Advanced Manufacturing Technology 2006, 28 (11-12), 1097-1103.

20. Packianather, M.; Griffiths, C.; Kadir, W., Micro injection moulding process parameter tuning. Procedia CIRP 2015, 33, 400-405.

21. Huang, C., Polymeric nanofeatures of $100 \mathrm{~nm}$ using injection moulding for replication. Journal of Micromechanics and Microengineering 2007, 17 (8), 1518.

22. Macintyre, D.; Thoms, S., The fabrication of high resolution features by mould injection.

Microelectronic Engineering 1998, 41, 211-214.

23. Pakkanen, T. T.; Hietala, J.; Pääkkönen, E. J.; Pääkkönen, P.; Jääskeläinen, T.; Kaikuranta, T., Replication of sub-micron features using amorphous thermoplastics. Polymer Engineering \& Science 2002, 42 (7), 1600-1608.

24. Schift, H.; David, C.; Gabriel, M.; Gobrecht, J.; Heyderman, L.; Kaiser, W.; Köppel, S.; Scandella, L., Nanoreplication in polymers using hot embossing and injection molding. Microelectronic Engineering 2000, 53 (1-4), 171-174.

25. Gadegaard, N.; Mosler, S.; Larsen, N. B., Biomimetic polymer nanostructures by injection molding. Macromolecular Materials and Engineering 2003, 288 (1), 76-83.

26. Yoshii, M.; Kuramoto, H.; Kato, K., Experimental study of transcription of minute width grooves in injection molding. Polymer Engineering \& Science 1994, 34 (15), 1211-1218.

27. Holthusen, A.-K.; Riemer, O.; Schmütz, J.; Meier, A., Mold machining and injection molding of diffractive microstructures. Journal of Manufacturing Processes 2017, 26, $290-294$.

28. Griffiths, C.; Dimov, S.; Scholz, S.; Tosello, G.; Rees, A., Influence of injection and cavity pressure on the Demoulding force in micro-injection moulding. Journal of Manufacturing Science and Engineering 2014, 136 (3), 031014.

29. Bellantone, V.; Surace, R.; Trotta, G.; Fassi, I., Replication capability of micro injection moulding process for polymeric parts manufacturing. The international journal of advanced manufacturing technology 2013, 67 (5-8), 1407-1421. 
30. Feldmann, M., The effects of the injection moulding temperature on the mechanical properties and morphology of polypropylene man-made cellulose fibre composites. Composites Part A: Applied Science and Manufacturing 2016, 87, 146-152.

31. Cunha, L.; Andritschky, M.; Pischow, K.; Wang, Z.; Zarychta, A.; Miranda, A.; Cunha, A., Performance of chromium nitride and titanium nitride coatings during plastic injection moulding. Surface and Coatings Technology 2002, 153 (2), 160-165.

32. Zhang, Y.; Hansen, H. N.; Sørensen, S., Replication of micro-pillars by PEEK injection moulding with $\mathrm{CrN}$-coated Ni tool. The International Journal of Advanced Manufacturing Technology 2015, 80 (14), 383-388.

33. Griffiths, C.; Bigot, S.; Brousseau, E.; Worgull, M.; Heckele, M.; Nestler, J.; Auerswald, J., Investigation of polymer inserts as prototyping tooling for micro injection moulding. The International Journal of Advanced Manufacturing Technology 2010, 47 (1-4), 111-123.

34. Yoon, S.-h.; Srirojpinyo, C.; Lee, J. S.; Mead, J. L.; Matsui, S.; Barry, C. M. In Evaluation of novel tooling for nanoscale injection molding, Smart Structures and Materials, International Society for Optics and Photonics: 2005; pp 107-116.

35. Menotti, S.; Hansen, H. N.; Bissacco, G.; Calaon, M.; Tang, P. T.; Ravn, C., Injection molding of nanopatterned surfaces in the sub-micrometer range with induction heating aid. The International Journal of Advanced Manufacturing Technology 2014, 74 (5-8), 907-916.

36. Kimura, F.; Kadoya, S.; Kajihara, Y., Effects of molding conditions on injection molded direct joining using a metal with nano-structured surface. Precision Engineering 2016, 45, 203-208.

37. Shen, Y.; Wu, W., An analysis of the three-dimensional micro-injection molding. International Communications in Heat and Mass Transfer 2002, 29 (3), 423-431.

38. Hopmann, C.; Behmenburg, C.; Recht, U.; Zeuner, K., Injection molding of superhydrophobic liquid silicone rubber surfaces. Silicon 2014, 6 (1), 35-43.

39. Stormonth-Darling, J. M.; Gadegaard, N., Injection moulding difficult nanopatterns with hybrid polymer inlays. Macromolecular Materials and Engineering 2012, 297 (11), 1075-1080.

40. Søgaard, E., Injection molded self-cleaning surfaces. 2014.

41. Stormonth-Darling, J. M.; Saeed, A.; Reynolds, P. M.; Gadegaard, N., Injection Molding Microand Nanostructures in Thermoplastic Elastomers. Macromolecular Materials and Engineering 2016, 301 (8), 964-971.

42. Su, Y.-C.; Shah, J.; Lin, L., Implementation and analysis of polymeric microstructure replication by micro injection molding. Journal of Micromechanics and Microengineering 2003, 14 (3), 415.

43. Zhang, N.; Srivastava, A. P.; Browne, D. J.; Gilchrist, M. D., Performance of nickel and bulk metallic glass as tool inserts for the microinjection molding of polymeric microfluidic devices. Journal of Materials Processing Technology 2016, 231, 288-300.

44. Zhang, N.; Srivastava, A.; Kirwan, B.; Byrne, R.; Fang, F.; Browne, D. J.; Gilchrist, M. D., Manufacturing microstructured tool inserts for the production of polymeric microfluidic devices. Journal of Micromechanics and Microengineering 2015, 25 (9), 095005.

45. Zhang, N.; Chu, J.; Byrne, C. J.; Browne, D.; Gilchrist, M., Replication of micro/nano-scale features by micro injection molding with a bulk metallic glass mold insert. Journal of Micromechanics and Microengineering 2012, 22 (6), 065019.

46. Puukilainen, E.; Rasilainen, T.; Suvanto, M.; Pakkanen, T. A., Superhydrophobic polyolefin surfaces: controlled micro-and nanostructures. Langmuir 2007, 23 (13), 7263-7268.

47. Kim, S.; Jeong, J.; Youn, J., Nanopattern insert molding. Nanotechnology 2010, 21 (20), 205302.

48. Kim, S. H.; Sul, I. H.; Jeong, J.-h.; Song, Y. S.; Youn, J. R., Robust fabrication and evaluation of nanopattern insert molded parts. European Polymer Journal 2013, 49 (6), 1437-1445.

49. Rogers, J. A.; Lee, H. H., Unconventional nanopatterning techniques and applications. John Wiley \& Sons: 2008.

50. Malek, C. K.; Coudevylle, J.-R.; Jeannot, J.-C.; Duffait, R., Revisiting micro hot-embossing with moulds in non-conventional materials. Microsystem technologies 2007, 13 (5-6), 475-481. 
51. Pfleging, W.; Hanemann, T.; Torge, M.; Bernauer, W., Rapid fabrication and replication of metal, ceramic and plastic mould inserts for application in microsystem technologies. Proceedings of the Institution of Mechanical Engineers, Part C: Journal of Mechanical Engineering Science 2003, 217 (1), 53-63.

52. Despa, M.; Kelly, K.; Collier, J., Injection molding of polymeric LIGA HARMs. Microsystem Technologies 1999, 6 (2), 60-66.

53. Lin, H.-Y.; Young, W.-B., Analysis of the filling capability to the microstructures in microinjection molding. Applied Mathematical Modelling 2009, 33 (9), 3746-3755.

54. Guo, Y.; Liu, G.; Zhu, X.; Tian, Y., Analysis of the demolding forces during hot embossing. Microsystem technologies 2007, 13 (5-6), 411-415.

55. Lin, H.-Y.; Chang, C.-H.; Young, W.-B., Experimental study on the filling of nano structures with infrared mold surface heating. International Polymer Processing 2011, 26 (1), 73-81.

56. Piotter, V.; Mueller, K.; Plewa, K.; Ruprecht, R.; Hausselt, J., Performance and simulation of thermoplastic micro injection molding. Microsystem Technologies 2002, 8 (6), 387-390.

57. Giboz, J.; Copponnex, T.; Mélé, P., Microinjection molding of thermoplastic polymers: a review. Journal of Micromechanics and Microengineering 2007, 17 (6), R96.

58. Rötting, O.; Röpke, W.; Becker, H.; Gärtner, C., Polymer microfabrication technologies. Microsystem Technologies 2002, 8 (1), 32-36.

59. Scholz, S.; Griffiths, C.; Dimov, S.; Brousseau, E.; Lalev, G.; Petkov, P., Manufacturing routes for replicating micro and nano surface structures with bio-mimetic applications. CIRP Journal of Manufacturing Science and Technology 2011, 4 (4), 347-356.

60. Becker, H.; Gärtner, C., Polymer microfabrication technologies for microfluidic systems. Analytical and bioanalytical chemistry 2008, 390 (1), 89-111.

61. Wang, Q. L.; Zhu, D. D.; Chen, Y.; Guo, X. D., A fabrication method of microneedle molds with controlled microstructures. Materials Science and Engineering: C 2016, 65, 135-142.

62. Schift, H.; Urwyler, P.; Kristiansen, P. M., Surface-patterned micromechanical elements by polymer injection molding with hybrid molds. Journal of Vacuum Science \& Technology B, Nanotechnology and Microelectronics: Materials, Processing, Measurement, and Phenomena 2013, 31 (6), 06FD01.

63. Michaeli, W.; Kamps, T., Micro assembly injection moulding with plasma treated inserts. Microsystem Technologies 2008, 14 (12), 1903-1907.

64. Pedersen, R. H.; Xu, Q.; Stormonth-Darling, J. M.; Gadegaard, N., Strategies for high quality injection moulding of polymer nanopillars. Macromolecular Materials and Engineering 2015, 300 (2), 172-180.

65. Ting, C.-J.; Huang, M.-C.; Tsai, H.-Y.; Chou, C.-P.; Fu, C.-C., Low cost fabrication of the largearea anti-reflection films from polymer by nanoimprint/hot-embossing technology. Nanotechnology 2008, 19 (20), 205301.

66. Hansen, T. S.; Selmeczi, D.; Larsen, N. B., Fast prototyping of injection molded polymer microfluidic chips. Journal of Micromechanics and Microengineering 2009, 20 (1), 015020.

67. Uhlmann, E.; Mullany, B.; Biermann, D.; Rajurkar, K.; Hausotte, T.; Brinksmeier, E., Process chains for high-precision components with micro-scale features. CIRP Annals-Manufacturing Technology 2016, 65 (2), 549-572.

68. Malek, C. G. K., Laser processing for bio-microfluidics applications (part I). Analytical and bioanalytical chemistry 2006, 385 (8), 1351-1361.

69. Malek, C. G. K., Laser processing for bio-microfluidics applications (part II). Analytical and bioanalytical chemistry 2006, 385 (8), 1362-1369.

70. Kong, J.; Xu, Y.; Yung, K.-L.; Xie, Y.; He, L., Enhanced polymer melts flow though nanoscale channels under vibration. The Journal of Physical Chemistry C 2008, 113 (2), 624-629.

71. Kim, E.; Xia, Y.; Whitesides, G. M., Micromolding in capillaries: applications in materials science. Journal of the American Chemical Society 1996, 118 (24), 5722-5731. 
72. Young, W.-B., Analysis of filling distance in cylindrical microfeatures for microinjection molding. Applied mathematical modelling 2007, 31 (9), 1798-1806.

73. Song, M.; Zhao, H.; Liu, J.; Liu, C.; Li, J., Replication of large scale micro pillar array with different diameters by micro injection molding. Microsystem Technologies 2016, 1-10.

74. Pranov, H.; Rasmussen, H. K.; Larsen, N. B.; Gadegaard, N., On the injection molding of nanostructured polymer surfaces. Polymer Engineering \& Science 2006, 46 (2), 160-171.

75. Yoo, Y.; Kim, T.; Choi, D.; Hyun, S.; Lee, H.; Lee, K.; Kim, S.; Kim, B.; Seo, Y.; Lee, H., Injection molding of a nanostructured plate and measurement of its surface properties. Current Applied Physics 2009, 9 (2), e12-e18.

76. Piotter, V.; Holstein, N.; Plewa, K.; Ruprecht, R.; Hausselt, J., Replication of micro components by different variants of injection molding. Microsystem Technologies 2004, 10 (6-7), 547-551.

77. Smith, D. E.; Tortorelli, D. A.; Tucker, C. L., Analysis and sensitivity analysis for polymer injection and compression molding. Computer Methods in Applied Mechanics and Engineering 1998, 167 (3-4), 325-344.

78. $\quad$ Xu, B.; Ooi, K.; Wong, T.; Liu, C., Study on the viscosity of the liquid flowing in microgeometry. Journal of Micromechanics and Microengineering 1999, 9 (4), 377.

79. Rosenbaum, E. E.; Hatzikiriakos, S. G., Wall slip in the capillary flow of molten polymers subject to viscous heating. AIChE journal 1997, 43 (3), 598-608.

80. Pirskanen, J.; Immonen, J.; Kalima, V.; Pietarinen, J.; Siitonen, S.; Kuittinen, M.; Mönkkönen, K.; Pakkanen, T.; Suvanto, M.; Pääkkönen, E., Replication of sub-micrometre features using microsystems technology. Plastics, rubber and composites 2005, 34 (5-6), 222-226.

81. Yang, C.; Li, L.; Huang, H.; Castro, J. M.; Yi, A. Y., Replication characterization of microribs fabricated by combining ultraprecision machining and microinjection molding. Polymer Engineering \& Science 2010, 50 (10), 2021-2030.

82. Sha, B.; Dimov, S.; Griffiths, C.; Packianather, M. S., Micro-injection moulding: factors affecting the achievable aspect ratios. The International Journal of Advanced Manufacturing Technology 2007, 33 (1-2), 147-156.

83. Miikkulainen, V.; Suvanto, M.; Pakkanen, T. A.; Siitonen, S.; Karvinen, P.; Kuittinen, M.; Kisonen, H., Thin films of MoN, WN, and perfluorinated silane deposited from dimethylamido precursors as contamination resistant coatings on micro-injection mold inserts. Surface and Coatings Technology 2008, 202 (21), 5103-5109.

84. Saha, B.; Toh, W. Q.; Liu, E.; Tor, S. B.; Hardt, D. E.; Lee, J., A review on the importance of surface coating of micro/nano-mold in micro/nano-molding processes. Journal of Micromechanics and Microengineering 2015, 26 (1), 013002.

85. Michaeli, W.; Klaiber, F.; Scholz, S., Investigations in variothermal injection moulding of microstructures and microstructured surfaces. Multi-Material Micro Manufacture 2008.

86. Lucchetta, G.; Sorgato, M.; Carmignato, S.; Savio, E., Investigating the technological limits of micro-injection molding in replicating high aspect ratio micro-structured surfaces. CIRP AnnalsManufacturing Technology 2014, 63 (1), 521-524.

87. Lucchetta, G.; Ferraris, E.; Tristo, G.; Reynaerts, D., Influence of mould thermal properties on the replication of micro parts via injection moulding. Procedia CIRP 2012, 2, 113-117.

88. Griffiths, C.; Tosello, G.; Dimov, S.; Scholz, S.; Rees, A.; Whiteside, B., Characterisation of demoulding parameters in micro-injection moulding. Microsystem Technologies 2015, 21 (8), 1677-1690.

89. Yokoi, H.; Han, X.; Takahashi, T.; Kim, W., Effects of molding conditions on transcription molding of microscale prism patterns using ultra-high-speed injection molding. Polymer Engineering \& Science 2006, 46 (9), 1140-1146.

90. Whiteside, B. R.; Martyn, M. T.; Coates, P. D.; Greenway, G.; Allen, P.; Hornsby, P., Micromoulding: process measurements, product morphology and properties. Plastics, rubber and composites 2004, 33 (1), 11-17.

91. Zhao, J.; Chen, G.; Chan, P.; Debowski, M. Investigation of interactions of polymer material, mould design and process condition in the micro moulding process; Citeseer: 2003. 
92. Su, Q.; Zhang, N.; Gilchrist, M. D., The use of variotherm systems for microinjection molding. Journal of Applied Polymer Science 2016, 133 (9).

93. Chen, M.; Yao, D.; Kim, B., Eliminating flow induced birefringence and minimizing thermally induced residual stresses in injection molded parts. Polymer-Plastics Technology and Engineering 2001, 40 (4), 491-503.

94. Michaeli, W.; Opfermann, D.; Kamps, T., Advances in micro assembly injection moulding for use in medical systems. The International Journal of Advanced Manufacturing Technology 2007, 33 (12), 206-211.

95. Sha, B.; Dimov, S.; Griffiths, C.; Packianather, M., Investigation of micro-injection moulding: factors affecting the replication quality. Journal of Materials Processing Technology 2007, 183 (2), 284296.

96. Zhao, J.; Mayes, R.; Chen, G.; Chan, P.; Xiong, Z., Polymer micromould design and micromoulding process. Plastics, Rubber and composites 2003, 32 (6), 240-247.

97. Yu, L.; Koh, C. G.; Lee, L. J.; Koelling, K. W.; Madou, M. J., Experimental investigation and numerical simulation of injection molding with micro-features. Polymer Engineering \& Science 2002, 42 (5), 871-888.

98. Yoon, S.-H.; Cha, N.-G.; Lee, J. S.; Park, J.-G.; Carter, D. J.; Mead, J. L.; Barry, C. M., Effect of processing parameters, antistiction coatings, and polymer type when injection molding microfeatures. Polymer Engineering and Science 2010, 50 (2), 411.

99. Sha, B.; Dimov, S.; Pham, D.; Griffiths, C. In Study of factors affecting aspect ratios achievable in micro-injection moulding, 4M2005 Conf. on Multi-material Micro Manufacture, Karlsrhue, 2005.

100. Surace, R.; Trotta, G.; Fassi, I.; Bellantone, V., The micro injection moulding process for polymeric components manufacturing. INTECH Open Access Publisher: 2012.

101. Yang, C.; Huang, H. X.; Castro, J. M.; Yi, A. Y., Replication characterization in injection molding of microfeatures with high aspect ratio: influence of layout and shape factor. Polymer Engineering \& Science 2011, 51 (5), 959-968.

102. Eringen, A. C.; Okada, K., A lubrication theory for fluids with microstructure. International journal of engineering science 1995, 33 (15), 2297-2308.

103. Heckele, M.; Schomburg, W., Review on micro molding of thermoplastic polymers. Journal of Micromechanics and Microengineering 2003, 14 (3), R1.

104. Gornik, C. In Injection moulding of parts with microstructured surfaces for medical applications, Macromolecular Symposia, Wiley Online Library: 2004; pp 365-374.

105. Chen, C.-P.; Chuang, M.-T.; Hsiao, Y.-H.; Yang, Y.-K.; Tsai, C.-H., Simulation and experimental study in determining injection molding process parameters for thin-shell plastic parts via design of experiments analysis. Expert Systems with Applications 2009, 36 (7), 10752-10759.

106. Nakao, M.; Tsuchiya, K.; Sadamitsu, T.; Ichikohara, Y.; Ohba, T.; Ooi, T., Heat transfer in injection molding for reproduction of sub-micron-sized features. The International Journal of Advanced Manufacturing Technology 2008, 38 (3), 426-432.

107. Song, M.; Liu, Z.; Wang, M.; Yu, T.; Zhao, D., Research on effects of injection process parameters on the molding process for ultra-thin wall plastic parts. Journal of Materials Processing Technology 2007, 187, 668-671.

108. Zhao, J.; Mayes, R.; Chen, G.; Xie, H.; Chan, P. S., Effects of process parameters on the micro molding process. Polymer Engineering \& Science 2003, 43 (9), 1542-1554.

109. Su, Q.; Gilchrist, M. D., Demolding forces for micron-sized features during micro-injection molding. Polymer Engineering \& Science 2016, 56 (7), 810-816.

110. Delaney, K.; Bissacco, G.; Kennedy, D., A structured review and classification of demolding issues and proven solutions. International Polymer Processing 2012, 27 (1), 77-90.

111. Berger, G. R.; Steffel, C.; Friesenbichler, W., A study on the role of wetting parameters on friction in injection moulding. International Journal of Materials and Product Technology 2016, 52 (1-2), 193-211. 
112. Wu, C. H.; Liang, W. J., Effects of geometry and injection-molding parameters on weld-line strength. Polymer Engineering \& Science 2005, 45 (7), 1021-1030.

113. Peng, Z.; Gang, L.; Yangchao, T.; Xuehong, T., The properties of demoulding of Ni and Ni-PTFE moulding inserts. Sensors and Actuators A: Physical 2005, 118 (2), 338-341.

114. Pontes, A.; Pouzada, A., Ejection force in tubular injection moldings. Part I: Effect of processing conditions. Polymer Engineering \& Science 2004, 44 (5), 891-897.

115. Pontes, A.; Pouzada, A.; Pantani, R.; Titomanlio, G., Ejection force of tubular injection moldings. Part II: A prediction model. Polymer Engineering \& Science 2005, 45 (3), 325-332.

116. Sasaki, T.; Koga, N.; Shirai, K.; Kobayashi, Y.; Toyoshima, A., An experimental study on ejection forces of injection molding. Precision engineering 2000, 24 (3), 270-273.

117. Correia, M. S.; Miranda, A. S.; Oliveira, M. C.; Capela, C. A.; Pouzada, A. S., Analysis of friction in the ejection of thermoplastic mouldings. The International Journal of Advanced Manufacturing Technology 2012, 59 (9), 977-986.

118. Beck, M.; Graczyk, M.; Maximov, I.; Sarwe, E.-L.; Ling, T.; Keil, M.; Montelius, L., Improving stamps for $10 \mathrm{~nm}$ level wafer scale nanoimprint lithography. Microelectronic Engineering 2002, 61, 441448.

119. Chou, S. Y.; Krauss, P. R.; Renstrom, P. J., Nanoimprint lithography. Journal of Vacuum Science \& Technology B: Microelectronics and Nanometer Structures Processing, Measurement, and Phenomena 1996, 14 (6), 4129-4133.

120. Christiansen, A. B.; Clausen, J. S.; Mortensen, N. A.; Kristensen, A., Injection moulding antireflective nanostructures. Microelectronic Engineering 2014, 121, 47-50.

121. Yoon, S.-H.; Lee, K.-H.; Palanisamy, P.; Lee, J.; Cha, N.-G.; Carter, D.; Mead, J.; Barry, C., Enhancement of surface replication by gas assisted microinjection moulding. Plastics, Rubber and Composites 2010, 39 (7), 293-299.

122. Schift, H.; Saxer, S.; Park, S.; Padeste, C.; Pieles, U.; Gobrecht, J., Controlled co-evaporation of silanes for nanoimprint stamps. Nanotechnology 2005, 16 (5), S171. 